EXEMPLARIa Classica

Journal of Classical Philology

21, 2017, pp. 175-215

ISSN 1699-3225

\title{
COMMENTER PROPERCE, UNE AFFAIRE COMPLEXE ET ÉMINEMMENT PHILOLOGIQUE
}

Paolo Fedeli, Irma Ciccarelli and Rosalba Dimundo, Properzio. Elegie, libro IV (2 vols.). Studia Classica et Mediaevalia, 7, Nordhausen: Verlag T. Bautz, 2015, 798 pp.; 735 pp., € 160.00, ISBN 978-3-88309-937-8.

Écrire 1527 pages, même aérées par une typographie qui en rend la lecture agréable, sur onze poèmes (le livre IV de nos éditions de Properce) qui, en tenant compte des pertes et des suppressions supposées par les commentateurs, totalisent 940 vers, voilà qui suscitera l'admiration de ceux qui, comme celui qui écrit ces lignes, n'ont pas la plume abondante et facile. Voici la répartition des vers par poème, en suivant le texte de Fedeli et de ses deux acolytes: $150+64+72+94+76+86+96+88+72+48+94$. Cette répartition fait immédiatement apparaître l'étendue extraordinaire de ce qui, d'après la tradition manuscrite, constitue une seule et même première élégie. P. Fedeli me paraît se fourvoyer certainement en soutenant qu'il n'y a pas là en réalité deux poèmes et que par conséquent le dernier livre comprend onze et non douze élégies. Sur ces douze poèmes, les pièces I (I A), III (II), V (IV), VII (VI), X (IX), XI (X) peuvent être considérées comme des "élégies romaines » en opposition à des élégies qu'on peut rapporter à un cycle érotique et/ ou pseudo-biographique qui met en scène une persona loquens nommée Propertius. Ces élégies sont les pièces II (IB), IV (III, une «Héroïde»), VI(V), VIII (VII), IX (VIII). La dernière élégie, qui fait parler la défunte Cornélie, me semble être à part. On constate un principe d'alternance entre élégies romaines et élégies du cycle érotique et/ou pseudo-biographique, si ce n'est que les pièces VIII (VII) et IX (VIII), consacrées à Cynthie, se suivent l'une l'autre. Je suggère que l'agenceur du recueil avait partout mis en ouvre le principe d'alternance et que le poème X (IX) relatif à la fondation de l'Ara Maxima devait originellement figurer entre les deux élégies dévolues à Cynthie. Je me permets de compléter par cette hypothèse et ces considérations celles que l'introduction de Fedeli consacre à la question de l'architecture du recueil. Bien sûr, le principe d'alternance n'exclut pas d'autres point de vues: ainsi, il est signifiant que la première moitié du recueil se termine avec la pièce $\mathrm{VI}(\mathrm{V})$, malédiction prononcée contre la défunte entremetteuse Acanthis, tandis que l'élégie funèbre qui donne la parole à la défunte Cornélie clôt l'ensemble du recueil. Soit dit en passant, cette frappante construction qui partage le recueil en deux moitiés égales corrobore l'idée que la première pièce représente en 
fait deux poèmes. Il est aussi signifiant que la pièce VIII (VII) est une élégie qui fait parler la défunte Cynthie, anticipation du discours d'outre-tombe de Cornélie sur fond d'opposition de caractère entre le personnage vindicatif et plein de rancœur qu'est Cynthie et la dignité apaisée et majestueuse de Cornélie. Il est remarquable, d'un point de vue "métapoétique », que la voix du poète s'éteigne non avec la mort de Cynthie, figure de la poésie propertienne, mais avec celle de la noble Cornélie, cette " anti-Cynthie ", modèle de devoir, de piété et de bienveillance, incarnation parfaite de la matrone romaine de haut lignage et de grande vertu. L'opposition entre " élégies romaines " et élégies du cycle érotique et/ou pseudo-biographique est relative, car les pièces VI (V), VIII (VII) et IX (VIII) contribuent à faire du livre IV un voyage poétique dans la topographie romaine archaïque et augustéenne.

Le commentaire (I, IV, VI, VIII, IX, XI Fedeli; III, V, VII Dimundo; II, $\mathrm{X}$ Ciccarelli) s'appuie sur un texte établi à partir de l'édition oxonienne de S. J. Heyworth (2007), à tous égards la plus importante édition critique de Properce depuis l'édition révolutionnaire d'Emil Baehrens (Leipzig 1880). Il n'y a pas, hélas, d'apparat critique mais une liste des passages où le texte de Heyworth et celui du trio italien different. Certain mais difficile à apprécier exactement semble être le rôle de l'édition radicalement non conservatrice de Heyworth dans l'évolution personnelle de Fedeli vers un conservatisme plus tempéré et ouvert aux constats d'une critique verbale un peu compétente. On peut prendre la mesure de ce changement en comparant au texte du commentaire de 2015 celui du commentaire de 1965 (Bari) ou de l'édition Teubner de 1984. Cette évolution montre que le cas des critiques conservateurs aux prises avec un texte profondément corrompu n'est pas toujours désespéré. Ma reconnaissance de l'importance éminente des mérites de la contribution ininterrompue de Fedeli aux études propertiennes rendra, je l'espère, plus vénielles les critiques que je m'apprête à formuler. L'évolution de Fedeli vers une critique moins conservatrice, assise sur le jugement juste, la perception exacte et fine des difficultés textuelles liées à la grammaire, à la phraséologie et au sens, n'est pas achevée et n'assure pas encore au vaste commentaire un degré suffisant de solidité et de fiabilité. Même si ici et là Fedeli et ses collègues marquent des points sur Heyworth, en particulier grâce à leur retenue en matière d'athétèse, trop de problèmes textuels sont encore soit non vus soit mal appréciés et mal réglés (ce n'est pas à dire que Heyworth ait bien réglé toutes les difficultés qu'il a vues). Le commentaire italien eût été meilleur si le texte de Properce eût été mieux transmis. Néanmoins, malgré la quantité de travail investie, de connaissances et d'érudition mobilisées, l'armature des commentateurs les laisse parfois impuissants même dans des passages que ne signale aucune difficulté liée à l'établissement du texte. L'intolérance à l'égard de la transposition conjecturale de vers n'a pas décru au point d'empêcher le maintien de l'ordre transmis de compromettre l'intelligibilité et la beauté de parties entières, voire d'un poème entier. La confusion qui caractérisait le 
répertoire grammatical de l'édition de 1984 s'est dissipée notablement, non entièrement. Tout cela sera illustré dans les notes qui suivent ${ }^{1}$. On voudra bien noter que leur but n'est pas de stigmatiser les défaillances des auteurs mais de contribuer à une plus juste appréciation des difficultés du texte de Properce, exceptionnelles par leur nombre et leur gravité. Les auteurs méritent tous les éloges pour la facilité de lecture que garantissent à leur commentaire la simplicité et la clarté élégantes avec lesquelles ils s'expriment. Ils procèdent par groupes de vers et mettent tant de liant entre les parties qu'on peut lire le commentaire entier de chaque élégie d'une seule traite et avec plaisir, la plupart du temps sans se lasser et très souvent sans s'interroger sur ce qu'ils ont voulu dire. On a l'impression d'un cours érudit, bien informé, très soigné, très bien rédigé, destiné à être compris et goûté au delà du cercle très restreint de quelques spécialistes chevronnés. Le style délayé s'oppose au style télégraphique parfois obscur de commentaires aux auteurs desquels on a fait l'obligation d'être brefs. Le revers de la médaille est que le ronron élégant d'une prose aimable risque de donner le change et de laisser au lecteur qui se laisse bercer l'impression que toutes les difficultés ont été sinon bien réglées, ce qu'on ne peut attendre d'aucun critique, si génial soit-il, du moins justement appréciées. L'œuvre qu'ont accomplie les trois auteurs n'est peut-être pas d'une originalité " décoiffante » mais, en tant que synthèse de l'érudition précédente, elle est en tout cas suffisamment importante pour devoir être consultée aussi par les critiques les plus avertis. Une bibliographie considérable, lue et maîtrisée, montre la qualité du travail préparatoire accompli par les commentateurs, et de très abondants index permettent de saisir, dans leur variété et aussi dans leurs limites, les richesses de leur commentaire. Je crois qu'il est instructif de le comparer, malgré la différence des textes, au commentaire d'Eduard Fraenkel à l'Agamemnon (1045 pages sur 1673 vers): ce que le commentaire sur Properce gagne en lisibilité, en légèreté, en agrément " littéraire », il le perd en minutie philologique par rapport au commentaire sur Eschyle. La minutie philologique extrême qui fait que le vaste commentaire d'une œuvre particulière est une source d'illumination pour tous les philologues classiques,

${ }^{1}$ Je regrette que Fedeli 191 persiste (il n'est pas le seul) à parler d'allongement métrique au frappé (arsis) à propos de I 10,23 neu si quid petiit (voir ma note à Valerius Flaccus VIII 67). Fedeli admet pourtant que IV 1,17 nulli cura fuit puisse être non un allongement métrique, mais un archaïsme, la quantité de -it au parfait étant originellement longue. La raison pour laquelle il admet ici la possibilité d'un archaïsme est que c'est, dit-il, l'unique cas certain chez Properce d'un allongement au frappé (affirmation répétée chez Dimundo 785): il oublie I 10,23, qu'il vient de présenter comme allongement au frappé "in coincidenza con la cesura ». Cette coincidence s'observe aussi en IV 1,17... Sur la question, voir, outre les travaux cités dans ma note à Valerius Flaccus, W. Corssen, Über Aussprache, Vokalismus und Betonung der lateinischen Sprache ${ }^{2}$, Leipzig II 1870, notamment 445-6 et 494. Corssen note que la graphie - eit s'est conservée jusqu'à l'époque augustéenne comprise et voit là la perduration du souvenir de la quantité primitive. Il se peut donc que la scansion iambique de fuit ne soit même pas un archaïsme! 
c'est peut-être ce qui manque au travail des trois érudits italiens. Mais seraitil juste de leur en faire reproche ? Je leur reprocherais plus facilement de jeter aux oubliettes, au profit d'une bibliographie récente, des travaux plus anciens capables de nourrir la réflexion ${ }^{2}$ et de ne pas avoir poussé assez loin les recherches en matière de realia. Évoquons ici un tout petit détail, puis un autre plus considérable. Teneros urit lorica lacertos, est-il dit (III 23) du malheureux Lycotas sous les armes: comment est-ce possible si « la lorica era il corpetto di cuoio o di metallo che serviva a proteggere petto e dorso " ? Le vers prouve qu'il existait des manches longues, qui d'ailleurs augmentent la pénibilité de la condition de Lycotas.

Les vers 12-13 (nec sine teste deo..., discuté plus bas) de l'élégie IX, étiologie de la fondation par Hercule lui-même de l'Ara Maxima sur le Forum Boarium, contiennent une allusion très discrète mais, me semblet-il, indiscutable à Iuppiter Inuentor, découvreur du vol des bœufs ${ }^{3}$. Hertzberg 1845 486-487, sensible aux aspects " antiquaires ", mentionne, dans son introduction au poème, Iuppiter Inuentor et l'autel qu'il est censé se voir consacrer, non loin de l'Ara Maxima, par Hercule pour lui avoir fait retrouver les bœufs. Il est remarquable que Properce n'évoque pas directement cet autel et qu'Hercule présente l'Ara Maxima comme gregibus deuota... repertis (67). Hertzberg remarque que ces mots, si on les interprète comme signifiant pro gregibus repertis uota (ainsi Fedeli 1186 !), pourraient faire allusion à l'autel de Iuppiter Inuentor. Mais, observe-t-il, Properce a en vue l'Ara Maxima et gregibus deuota repertis doit signifier dicata postquam greges repperi. Sans s'interroger sur le sens des mots de Properce, Hutchinson 2006218 affirme que, comme Ovide Fastes I 579-582, Properce confond (" conflates") les deux autels 4 . Mais, selon Hertzberg, chez Ovide, les vers 579-580 sont relatifs à l'autel de Iuppiter Inuentor, les vers 581-582 à l'Ara Maxima. Si, comme il me semble, Hertzberg a raison ${ }^{5}$, il y a lieu de croire que Properce ne confond pas non plus les deux autels: il fait bien allusion à Iuppiter Inuentor mais a choisi de ne pas évoquer directement l'autel que lui consacra Hercule. Je n'exclus toutefois pas que Properce ait joué sur l'ambiguïté des mots gregibus deuota repertis: pris dans un sens, ils désignent l'Ara Maxima et, dans un autre, l'autel de Iuppiter Victor. Mais cette « conflation » bien spéciale n'implique aucune confusion. Relevons aussi

${ }^{2}$ Il y a là un trait qui oppose au commentaire de Fraenkel la plupart des commentaires qui paraissent aujourd'hui.

${ }^{3}$ Voir M. Bréal, Hercule et Cacus. Étude de mythologie comparée, Paris 1863, 45-6, 49; G. Wissowa, Religion und Kultus der Römer, Munich 1912², 273 et 275.

${ }^{4}$ Ils sont bien distincts: voir J. Carcopino (compte rendu de J. Bayet, Les origines de l'Hercule romain Paris 1926), JS 5, 1928, 206.

${ }_{5}^{5}$ S. J. Green, auteur du dernier commentaire sur le premier livre des Fastes (Leyde 2004, $267)$, ne se pose pas la question de savoir si les vers $579-580$ sont relatifs à cet autel. J. G. Frazer et F. Bömer ne s'interrogent pas non plus. 
que, en faisant dire à Hercule Fontis egens erro circum antra sonantia lymphis (35, avec circum antra de Burman pour circaque $e^{6}$, Properce paraît faire allusion à une étymologie $\left(\pi \varepsilon \imath v \tilde{\omega} v \tau \varepsilon \varsigma^{7}\right) \mathrm{du}$ nom des fameux Pinarii, qui avaient en charge d'assister l'officiant du culte de l'Ara Maxima. C'est dire que, pour faire justice à Properce, il faut être aussi subtil que lui - mais non plus subtil que lui en le surinterprétant, ce que j’espère ne pas avoir fait.

\section{VERS PERDUS ET INTERPOLÉS}

La seule perte ${ }^{8}$ admise dans ce livre d'élégies par les auteurs, qui suivent ici Heyworth, est intervenue entre Iuppiter et obliquae signa iterata rotae (I B 82): ont donc disparu un hexamètre intermédiaire et les portions de deux pentamètres. D'après le texte transmis de I A 19-20, annuaque accenso celebrare (celebrata Fedeli d'après Phillimore, celebrante Housman) Parilia faeno, qualia nunc curto lustra nouantur equo, aux antiques Parilia (A) du 21 avril serait comparé un rituel moderne (qualia nunc) de purification lié à un equus curtus (B), qui semble être le fameux October equus. Cette comparaison paraît absurde; selon toute apparence, A et B sont également archaïques. Fedeli admet que curto lustra nouantur equo vise un rituel nouveau, différent de celui du Cheval d'Octobre, mais - pour ne pas insister sur l'invraisemblance intrinsèque de l'idée que curto equo pourrait se rapporter à autre chose qu'au cheval amputé de sa queue du rituel ancien - qualia nunc curto lustra nouantur equo signifierait alors " rites de purification semblables à ceux qui sont aujourd'hui inventés avec la mutilation d'un cheval ». Mais le sens très nettement plus plausible, à mon avis, serait «rites lustraux semblables à ceux qui sont renouvelés avec la mutilation du cheval». Or qualia nunc devrait rapprocher, tout en les opposant, un rituel ancien et un rituel nouveau. On arrive donc à une aporie. D'où mon idée qu'il y eut perte entre qualia nunc et la suite:

qualia nunc

curto lustra nouantur equo.

Heyworth (Cynthia 417) rejette toute comparaison avec l'époque contemporaine du locuteur et considère 19-20 (avec la leçon celebrare) comme interpolés, mais qui aurait pu concevoir un distique de ce genre, où notamment curto lustra nouantur equo paraît se rapporter avec une allusivité érudite à la fête de l'October equus (Fedeli 196-197) ? L'hypothèse

${ }^{6}$ Circ $<a$ aru $>a$ (Baehrens) est plus proche.

${ }^{7}$ Voir Carcopino 214-215.

${ }^{8} \mathrm{Je}$ ne tiens pas compte des pertes causées par la substitution aux parties originales de morceaux interpolés ou répétés par erreur. 
de l'interpolation est, si je ne m'abuse, la moins plausible de toutes; moins invraisemblable serait l'idée d'une corruption de qualia nunc. Mais je préfère l'hypothèse d'une omission. Quoi qu'il en soit, tel qu'il est transmis, le texte des v. 19-20 ne se tient pas. La difficulté échappe à Fedeli.

Les seules interpolations que retiennent les commentateurs italiens sont celles de I B 87-88, 125-126; V 55-56, IX 73-74 et XI 39-40. Fedeli rejette, tel qu'il est, IX 42: c'est, à proprement parler, une anticipation du v. 66 à l'époque où le distique 65-66 suivait immédiatement 41-42. L'anticipation a provoqué l'omission de 65-66, réintroduit dans le texte au mauvais endroit, après le distique qui commence par $A t(63)$ et non après celui (41-42) qui commence par Atque. Cette explication de la faute me semble plus simple que celles que mentionne Fedeli 1167. 22 vers séparent les vers $65-66$ du vers qui les précédait primitivement: nous reviendrons bientôt sur ce chiffre. Il est clair que IX 7172 sont censés clore le poème (Sance, uelis libro dexter inesse meo), et on obtient cette clôture soit en supprimant 73-74 (Richardson 1977, suivi par Fedeli) soit en le transposant avant 71-72. Mais la véritable conclusion du poème étiologique pourrait bien être le distique 69-70: Amphitryoniades (mot initial du poème) et Herculis (mot initial du dernier, suivi d'une épithète corrompue dans la tradition) se répondent. Si l'épithète de Herculis indiquait bien, comme nous le verrons plus bas, le statut d'exclu et que le poète renvoyât ainsi au statut d'amator exclusus de son propre personnage, le caractère conclusif du vers 70 est d'autant plus frappant. Le vers 72 forme d'ailleurs une conclusion peut-être plus adaptée à la fin d'un recueil (libro... $m e o)^{9}$ qu’à la fin de la pénultième pièce. On pourrait arguer que Properce luimême a mis cette conclusion dans l'intention de faire de cette pièce la dernière d'un recueil, mais je ne crois pas à cette hypothèse: j'ose suggérer qu'un lecteur érudit de l'Antiquité, trouvant trop abrupte la conclusion mise dans la bouche d'Hercule lui-même $\mathrm{e}^{10}$, aura voulu en mettre une plus nette (cf. salue) dans la bouche du poète: il se sera pour cela inspiré de Virgile, Én. VIII 301-302, que l'on cite pour illustrer 71-72 $2^{11}$. Mais l'introduction de Sance - si l'on admet cette correction de la leçon sancte - était brutale et le lien avec Hercule obscur: l'interpolation de 71-72 $2^{12}$ aura entraîné celle de 73-74. L'ordre voulu

${ }^{9}$ Comparer le dernier vers des Fastes d'Ovide, adnuit Alcides increpuitque lyram, avec les réflexions d'A. Barchiesi, The Poet and The Prince: Ovid and Augustan Discourse, Berkeley 1997, 268-9. Selon lui, le vers d'Ovide « sounds like a delayed answer to Propertius' prayer for his book of aetiological elegies ». A mon avis, l'allusion intertextuelle est imaginaire.

${ }^{10}$ L'élégie IV du livre I se termine sur une déclaration de Cynthie.

${ }^{11}$ On invoque aussi la clôture hymnologique avec $\chi \alpha \mathbf{i} \rho \varepsilon$ (salue) et Callimaque Aetia fr. 23,19-20 Pfeiffer, où Karl Dilthey (De Callimachi Cydippa, Leipzig 1863, 106) voulut voir, d'après Properce 71-72 transposé après 73-74, la fin d'un récit relatif à Héraclès. Par une singulière intuition, Dilthey (que ne citent ni Pfeiffer 32 ni Annette Harder, Callimachus Aetia, Oxford 2012, I, 157), se fondant sur la répétition de sancte et la prière contenue dans Properce 71-72, a deviné que le distique de Callimaque en était suivi d'un autre.

${ }^{12}$ Hypothèse formulée par U. Knoche dans "Gedanken zur Interpretation von Properzens 
était 73-74-71-72, mais la transmission aura interverti les distiques. Je ne suis pas certain que les corrections Sance (72) et Sancum (74) soient justes, car les interpolateurs présumés ont pu connaître l'emploi ${ }^{13}$ de Sanctus pour Sancus. Je n'exclus pas que l'auteur des v. 71-72, Properce ou un autre, ait écrit deux fois sancte en pensant non à Semo Sancus mais à Hercule admis parmi les dieux (71 sancte pater, salue, cui iam fauet aspera Iuno = Virgile Én. VIII 301 salue, uera Iouis proles, decus addite diuis) et que l'auteur des v. 7374 ait mal interprété Sancte en le rapportant à Semo Sancus. Rien, dans les vers 71-72, ne prouve que leur auteur ait eu en vue Semo Sancus et les érudits qui éliminent 73-74 (ainsi Fedeli) devraient s'aviser qu'ils interprètent 71-72 en fonction du distique qu'ils éliminent ! L'auteur des v.73-74 était peut-être maladroit ${ }^{14}$, mais il n'était pas inculte: il connaît l'équation Semo Sancus = Dius Fidius $=$ Héraclès $^{15}$ et la version liant à Titus Tatius la fondation du culte de Sancus ${ }^{16}$. Toutefois, il ne faudrait pas tirer argument de ce savoir pour contester l'hypothèse de l'interpolation, car la version exposée par l'auteur de 73-74 était, selon Wissowa, " am gelaüfigsten ". Ne serait-il pas étonnant qu'un poème relatif à l'étiologie de la fondation de l'Ara Maxima dans le Forum Boarium se termine par l'évocation d'un culte, celui de Semo Sancus, sis au Quirinal ${ }^{17}$ ? L'interpolateur semble d'ailleurs avoir raté l'occasion de mettre en valeur un lien qui unit Sancus, gardien des contrats et traités, à l'Ara Maxima: " c'est là qu'on venait jurer, sous la voûte du ciel, un silex à la main, les contrats les plus sacrés $»^{18}$.

Gedicht II 28”, Miscellanea Properziana, Assisi 1957, 69 n. 2 = Ausgewählte Kleine Schriften, Francfort 1986, 334 n. 2. Fedeli et Heyworth ignorent cette hypothèse, Hutchinson la mentionne sans la commenter. L'idée de Knoche est que 71-72 ont été conçus comme un substitut de 73-74. Il remarque que salue n'est pas ailleurs chez Properce.

${ }^{13}$ Wissowa, article "Sancus" du lexique de Roscher IV 316.

${ }^{14}$ Heyworth corrige peut-être l'interpolateur en substituant monstris au gauche manibus dans quoniam manibus purgatum sanxerat orbem. Purgatum sanxerat me parait en l'occurrence un délayage malheureux, bien que ce pléonasme (verbe conjugué à un mode personnel et participe passé passif adjectif qualifiant le substantif complément et offrant un sens voisin de celui du verbe conjugué) ne soit pas rare dans la poésie latine, comme nous l'avons rappelé dans notre commentaire critique sur les Silves (note à I 2,77 edomui uictum, <in>uictum Shackleton Bailey d'après Eden).

${ }^{15}$ Wissowa, Religion und Kultus der Römer , 130.

${ }^{16}$ Wissowa, article «Sancus » du lexique mythologique de Roscher IV 317.

${ }^{17}$ Voir, outre Wissowa, E. Norden, Aus altrömischen Priesterbüchern, Lund $1939=$ Stuttgart/Leipzig 1995, 209ss.

${ }_{18}$ Bréal, Hercule et Cacus, 48; voir aussi, par exemple, O. Gilbert, Geschichte und Topographie der Stadt Rom im Altertum I, Leipzig 1883, 75-7. Selon Bréal, c'est Zeus/Jupiter (gardien des serments) qui se cache sous (Semo) Sancus. Dans sa dissertation brillante Quaestiones mythologicae, Bonn 1869, 40, le jeune Hermann Osthoff préfère voir avec Varron, derrière l'Hercule de l'Ara Maxima, le dieu Mars, qu'il reconnaît aussi sous Semo Sancus. Il fait valoir que le culte de l'Hercule de l'Ara Maxima était placé sous la responsabilité des Saliens, qui s'occupent de Mars, et que les Semones accompagnent Mars dans le Carmen Aruale. 
En XI 39-40 Fedeli, habituellement plus rétif à cette médecine, a cédé (à tort, je crois) à la tentation de l'athétèse, à laquelle Heyworth n'a que partiellement résisté en remplaçant le vers 39, et Persen proaui stimulantem pectus Achilli, par un moignon, et <...................................., et illum>, qui tumidas (Heyworth, empruntant tumidas à Heyne; quique tuas mss.) proauo fregit Achille domus. Cornélie évoque Paul Émile, père de Scipion Émilien et vainqueur de Persée. Heyworth et Fedeli rejettent le texte conjectural admis par G. P. Goold (Loeb, 1999²):

et, Persen proaui stimulat dum pectus Achilli, qui tumidas proauo fregit Achille domos

« and him, who, when Perses was spurred on by the spirit of his ancestor Achilles, crushed the house inflated by its ancestor Achilles ". Heyworth (Cynthia 511) et Fedeli 1333 critiquent à juste titre la place de dum et de qui et s'offensent de la répétition proaui Achilli/proauo Achille: "without obvious rhetorical point "; « l'intollerabile poliptoto (...), in difesa del quale non si è portato alcun esempio valido in Properzio ". S'il n'y a que les objections grammaticales, on peut envisager, en combinant plusieurs corrections déjà proposées,

qui Persen, proaui stimulat quem pectus Achilli, et tumidas proauo fregit Achille domos.

Quid de l' "intolérable polyptote ", de la répétition "sans pertinence évidente " ? Cette répétition tempérée de uariatio ne me paraît nullement dépourvue de force rhétorique: de son ascendance achilléenne Persée tire individuellement la bravoure téméraire qui l'anime, de l'ascendance achilléenne la maison à laquelle Persée lui-même appartient tire collectivement sa superbe; Paul Émile brise Persée et sa maison, la témérité du premier, la superbe de la seconde. Au risque de choquer Heyworth et Fedeli, je ne trouve pas ladite répétition beaucoup moins efficace que Sit licet et saxo patientior illa Sicano, sit licet et ferro durior et chalybe (I 16,29-30), même si l'on a tenté ici aussi d'éliminer la reprise de sit licet ${ }^{19}$. Ce n'est donc pas de Silius Italicus XIV 94-95 Pyrrhus origo dabat stimulos proauique superbum | Aeacidae genus atque aeternus carmine Achilles et XV 292 proauoque tumebat Achille qu'un interpolateur aurait tiré le distique de Properce, mais c'est bien plutôt ce distique qui aurait inspiré Silius. Je suis d'accord avec

${ }^{19}$ Je renverrais avec plus de conviction à la partie de l'essai de Vahlen consacrée à la répétition chez Properce et d'autres poètes (Opuscula academica I, Leipzig 1908, 348-60) s'il faisait mieux la différence entre passages sains et passages corrompus. D. R. Shackleton Bailey, Propertiana, Cambridge 1956,9) ajoute d'autres passages en ne montrant pas plus de discernement que Vahlen. 
les autres athétèses pratiquées par Fedeli. En I B 125-126, l'interpolateur présumé s'est inspiré de I A 65-66, scandentes si quis cernet de uallibus arces, I ingenio muros aestimet ille meo, après la corruption de ingenium muris aestimet ille meum, si du moins cette conjecture de Markland, non mentionnée par Fedeli, rencontre le vrai.

\section{VERS DÉPLACÉS}

L'ordre de succession des vers est un des sujets les plus polémiques des études propertiennes. D'aucuns cherchent à justifier l'ordre transmis par une esthétique du "beau désordre ", du flux et du reflux censés caractériser la "Ringkomposition »; ils objectent à leurs contradicteurs que les déplacements qu'ils supposent sont - prétendument ! - inexplicables sauf à imaginer un mauvais génie qui se serait plu à perturber très gravement la succession originale des distiques et parfois à permuter des hexamètres ou des pentamètres. Comme à propos des conjectures, ils opposent que la multiplicité des déplacements proposés démontre leur inanité, comme si la pluralité de diagnostics posés par les médecins aux prises avec un cas complexe montrait la bonne santé du patient. On se trouve devant un dilemme: de la solution de continuité idiosyncrasique ou de la perturbation répétée de l'ordre original, laquelle est la plus plausible ? L'évolution de Fedeli, qui rejette dans le présent commentaire l'ordre transmis beaucoup plus souvent que dans son édition de 1984, est intéressante. Il accepte suffisamment de corrections de cet ordre pour que j'ose me demander si, une fois admise l'importance de la perturbation de la succession des vers dans la tradition textuelle de Properce, il n'est pas permis d'aller plus loin que lui quand on a des raisons sérieuses de mettre en doute l'ordre transmis. Prenons l'exemple de l' "Élégie de Vertumne » (II). Ciccarelli y conserve intégralement l'ordre des vers transmis, ce qui me paraît défigurer l'ensemble du poème, bien prêt d'être réduit à un ramas de membra disiecta. L'évocation de Rome v. 49-56 serait à sa place après le v. 4, où, en partie d'après Housman, O. L. Richmond les transpose. Cette transposition, entre autres bienfaits, rend intelligible haec (v. 5): Haec me turba iuuat nec templo laetor eburno: Romanum satis est posse uidere Forum. La chute des v. 49-56 pourrait s'expliquer par le phénomène de parablepsie: l'œil d'un copiste serait passé de Roma (v. 49) à Romanum (v. 6); les vers omis, ajoutés dans une marge, auraient été recopiés au mauvais endroit. Les vers 49-56 se trouvent séparés de leur vraie place par 44 vers: on retrouve ici en quelque sorte le chiffre de 22 vers déjà noté à propos du déplacement de IX 65-66. Là où ils sont, les vers $41-48$ de l' "élégie de Vertumne " créent une solution de continuité: le nam qui les introduit n'a pas de pertinence et le dernier distique est privé du développement (23-40) sur les métamorphoses de Vertumne qu'il appelle. Schrader est le premier à avoir suggéré la transposition de 41-46 après le v.18, et je crois que cette idée est très heureuse. L'œil d'un copiste aura glissé 
de fama v. 41 à fama v. 1920. Là encore ce sont 22 vers qui séparent de leur vraie place les v. 41-46. Une place possible pour le distique 47-48 est après le v. 20:

$\begin{array}{cl}\text { Mendax fama, uaces: †alius } \dagger \text { mihi nominis index: } & 19 \\ \text { de se narranti tu modo crede deo. } & 20 \\ \text { At mihi, quod formas unus uertebar in omnes, } & 47 \\ \text { nomen ab euentu patria lingua dedit. } & 48 \\ \text { Opportuna mea est cunctis natura figuris: } & 21 \\ \text { in quamcumque uoles uerte, decorus ero. } & 22\end{array}$

Cette transposition me paraît évidemment préférable à celle de Richmond, qui place $47-48$ après 22 . J'imagine que l'omission de $47-48$ est due au retour de nomen au v. 48 après nominis v. 19 . Heyworth procède à un remaniement trop compliqué que je ne veux pas discuter ici. Au v. 19, Ciccarelli 442 croit que alius mihi nominis index peut signifier « ben altro è il senso del (mio) nome " mais Heyworth me paraît avoir raison d'adopter la correction de Lachmann falsa es, c'est-à-dire falsa's.

Dimundo 573 défend faiblement le maintien à leur place dans les mss. des v. 55-56 de l'Héroïde (III), transférés par Housman après le v. 32, où ils conviennent parfaitement. La cause de la chute est l'homéotéleute laboro (v. 33) / una toro (v. 56). Là aussi un distique est séparé du passage qui le précédait originellement par 22 vers. Fut-ce le nombre de vers par page ou par colonne d'un ancêtre de la tradition textuelle ? Je dois me contenter ici de poser la question. En l'état du texte imprimé par Dimundo, la transition de V 20 à ce qui suit est problématique et il peut manquer des vers (Guyet). Dimundo écarte toute transposition de IV 29-30 et n'admet pas l'hypothèse (Heyworth) qu'il manque des vers avant le v. 29; en tout cas, elle a tort de refuser la correction de uirum (29) en moram. Me paraissent s'imposer la transposition de VI $45-46^{21}$ après 52 (Housman; cause présumée de la chute: l'écho regia uela v. 46 / Iulia rostra v. 54) et celle de VII 47-48 après 40 (Schrader; cause présumée de la chute: homéotéleute humum v. 40 / aurum v. 47). La transposition de VIII 9-10 après 11-12 (Housman), acceptée par Goold, ne l'est plus par Heyworth et Fedeli: ce n'est pas un progrès. DemissAE... mANVS et castAE... ANNVS pourraient être responsables de l'omission du premier distique ensuite ajouté à la mauvaise place. En ce qui concerne X 25-26, l'athétèse, suggérée par Heyworth et acceptée par Hutchinson, paraît interdite par la composition du poème, qui corrobore la transposition à l'intérieur de l'ensemble (5-22) relatif à l'histoire de Romulus

${ }^{20}$ Autre hypothèse chez O. L. Richmond, Sexti Properti quae supersunt opera, Cantabrigiae 1928, 337.

${ }^{21}$ Heyworth et Fedeli ont tort de ne pas considérer prope (45) comme corrompu.

ExClass 21, 2017, 175-215 
et Acron. En effet, avec la transposition de Passerat (5-22, 25-26) ou celle de Richmond (1-8, 25-26, 9-22), l'histoire de Romulus et Acron compte vingt vers, tandis que l'histoire de Cossus et Tolumnius (23-24, 27-38, soit 14 vers) et l'histoire de Claudius et Virdomar (39-44, six vers) totalisent vingt vers. La composition du poème est transparente: $1-4$ (prologue), $5-44$ (trois histoires réparties sur $2 \times 20$ vers, la seconde vingtaine étant partagée), 45-48 (conclusion). Pour ce qui est de XI 9-10, je ne sais, de la transposition ou de l'athétèse, laquelle est préférable; en tout cas, ce distique pose problème. Il est peut-être non seulement supposé (suppositicius) mais aussi altéré. En l'état du texte transmis, tibi dans Nunc tibi commendo, communia pignora, natos (XI 73) n'a pas de référent et $\mathrm{J}$. Butrica ${ }^{22}$ remédiait à cette difficulté en substituant Paulle à natos, apparemment imité par l'auteur de l'Alcestis Barcinonensis 95, ante omnes commendo tibi, pia pignora, natos. C'est apporter à un vrai problème une solution fausse. Le transfert de 81-84 après le v. 72 me paraît la véritable solution:

Haec est feminei merces extrema triumphi, $\quad 71$ laudat ubi emeritum libera fama †rogum $\dagger$. $\quad 72$ Sat tibi sint noctes quas de me, Paulle, fatiges, $\quad 81$ somniaque $\dagger$ in faciem credita saepe $\dagger$ meam: $\quad 82$ atque ubi secreto nostra ad simulacra loqueris, $\quad 83$ ut responsurae singula uerba iace. $\quad 84$ Nunc tibi commendo, communia pignora, natos. $\quad 73$

Fedeli défend rogum mais c'est torum (Koppiers) qui convient. Au v. 82, il met les croix autour de credita saepe seulement et, comme Heyworth et Hutchinson, semble ne pas connaitre ma proposition effigiem credita ferre (RPh 762002 71; pour la phraséologie, uisa referre serait meilleur). Le lecteur sans préjugé qui lira l'ensemble du passage avec cette transposition ne manquera pas, j'en suis sûr, de constater combien elle améliore le texte transmis. La raison de la chute des v. 81-84 est le saut de l'œil d'un copiste de tibi v. 81 à tibi v. 73.

\section{Ponctuation}

Il y a là un aspect encore trop souvent négligé mais important du travail critique et exégétique. Fedeli conserve la ponctuation traditionnelle en I A 9-10, Qua (quo ou quod les mss. autorisés) gradibus domus ista Remi se sustulit, olim | unus erat fratrum maxima regna focus. Il paraît peu douteux qu'ici, comme dans ce qui précède et ce qui suit, le passé et le présent sont opposés l'un à l'autre. Mais se sustulit ne saurait guère exprimer le présent opposé au passé et il ne sert à rien d'alléguer, comme fait Fedeli à la suite d' E.

${ }^{22}$ J. Butrica, The Manuscript Tradition of Propertius, Toronto 1984, 201. 
Wistrand ${ }^{23}$, des passages où le parfait exprime un état présent puisqu'ici on a besoin d'un contraste pareil à celui que font, dans ce qui précède et suit, uides, est / fuit; stant / procubuere; nitet / habuit. Le Nunc gradibus domus ista Remi se sustulit de Heyworth est très maladroit. Goold me semble avoir eu raison d'adopter la ponctuation géniale de W. S. Watt, Qua gradibus domus ista (c'est-à-dire, présume-t-on ${ }^{24}$, aedes Quirini, avec se tollit tiré de sustulit), Remi (c'est-à-dire la hutte de Romulus, prétendument remplacé par Rémus metri causa) se sustulit olim ${ }^{25}$ : | unus erat fratrum maxima regna focus. Fedeli 178 objecte que cette interprétation implique l'existence (je dirais plutôt « l'existence supposée par Properce ou sa source ") d'une casa Romuli (Quirinal) qui ne coïncide avec aucune des deux connues (Palatin et Capitole). Vu l'équation Quirinus $=$ Romulus ${ }^{26}$, serait-il si étonnant qu'on ait imaginé que les aedes Quirini reconstruites par Auguste et dédicacées par lui en 16 av. J.-C. soient le lointain successeur d'une primitive domus Romuli ? Mais, dans le passage tel que le ponctue Watt, domus ista ne renvoie pas nécessairement aux aedes Quirini: ne s'agit-il pas de ce que Martial XII 2,6 (édition Loeb de Shackleton Bailey) appelle domus alta Remi, c'est-àdire le complexe incluant la domus Augusti, le temple d'Apollon Palatin et la Bibliothèque du Palatin ${ }^{27}$ ? Les objections formulées par Fedeli 180-181 ne me paraissent nullement dirimantes. Le fait que les gradins (gradibus) pourraient être ceux du temple d'A pollon Palatin ${ }^{28}$, dont il a déjà été question v. 3-4, ne constitue pas, à mon sens, un obstacle. Fedeli préfère rapporter le vers de Properce à la réfection de la casa Romuli sur le Palatin, mais il faut quelque chose de plus significatif, de plus marquant, de plus imposant que cette obscure réfection. En l'état, le pentamètre unus erat fratrum maxima regna focus, " a single hearth was the total realm of the brothers " d'après la traduction arrangeante de Goold, est absurde, car Properce n'a pu ni penser ni dire que l'autorité royale de Romulus se limitait à son foyer. Le sens impose virtuellement regia magna, belle conjecture de L. Havet, dont on lira les remarques dans ses Notes critiques sur Properce, Paris 1916, 106-729. Ainsi,

${ }^{23}$ La leçon de linguistique que E. Wistrand, Miscellanea Propertiana, Göteborg 1977, 72-3 donne à Watt et aux latinistes " who tend to disregard the fact that the Latin perfect tense can denote a state produced by a preceding action that is hardly present to the mind ", est à côté de la plaque.

${ }^{24}$ D'après le commentaire de M. Rothstein, Berlin $1924^{2}$ II, 191 et 378 (corrige la note de l'édition de 1898).

${ }^{25}$ Comparer par exemple XI 79, Et si quid doliturus eris sine testibus illis, c'est-à-dire si quid doliturus eris, <id doleto $>$ sine testibus illis.

${ }^{26}$ Voir par exemple W. Weber, Princeps (I), Stuttgart/Berlin 1936, 86* n. 398.

${ }^{27}$ Voir J. Beaujeu, Mélanges P. Boyancé, Rome 1974, 57-72.

${ }^{28}$ Beaujeu, Mélanges, 70, préfère « la Scala Caci montant de la Vallée du Grand Cirque à la plate-forme nord-ouest du Palatin ".

${ }^{29}$ Rothstein semble avoir perçu le vrai sens mais, bien qu'intelligent, il était outrageusement conservateur et paraît avoir cru que maxima regna pouvait signifier regia magna. Havet a raison de l'en blâmer. Beaujeu 72 n. 2 et Fedeli 182, dont les remarques sur le passage sont des 
le distique oppose de deux manières complémentaires l'antique foyer de Romulus et Rémus et le complexe monumental du Palatin contemporain du locuteur. Quoi de plus approprié ? Il y a, si je ne m'abuse, dans la ponctuation de Watt une des plus belles pièces à ajouter à l'essai justement célèbre de Vahlen de distinctionis usu critico (Opuscula academica I 103-120). Aux v. 13-14 de l'élégie IV, Murus erat montes: ubi nunc est Curia saepta, | bellicus e uiuo (e uiuo Waardenburgh: ex illo mss.) fonte bibebat equus, Fedeli 618619 récuse la ponctuation et l'interprétation ubi nunc est Curia, saepta erant, mais saepta épithète qualifiant Curia fait difficulté. La ponctuation récusée donne à ex illo (mss.) la pertinence qui lui manque. Au contraire de Fedeli 2015, Fedeli 1965 et 1984 acceptait ex illo et la ponctuation qui fait de saepta le sujet d'un verbe sous-entendu. Si Fedeli se range à l'analyse que donne Vahlen de VI 63-64, Illa petit Nilum cumba male nixa fugaci, I hoc unum, iusso non moritura die, Dimundo ne rend pas justice à l'explication par Vahlen de VII 29-30 et Fedeli aux observations du même sur XI 3536. Dans le premier passage, Dimundo garde le texte transmis Si piguit portas ultra procedere, at illuc|iussisses lectum lentius ire meum. Mais illuc, qui est sans référent exprimé, fausse le tour lectum ire, où ire est employé absolument d'une manière idiomatique comme dans l'expression funus it. Il est regrettable que la correction de Muret et d'Heinsius illud, que développe idiomatiquement ${ }^{30}$ l'infinitive lectum lentius ire meum, soit à ce point négligée par la critique récente. Pour ce qui est de XI 35-36, Fedeli semble avoir raison d'adopter la correction de Graevius ut pour in: iungor, Paulle, tuo sic discessura cubili, | ut lapide hoc uni nupta fuisse legar. Fedeli condamne l'interprétation de Vahlen selon laquelle l'infinitive uni nupta fuisse développe hoc (hoc legar ne serait que la tournure passive correspondant à hoc legent sc. me uni nuptam fuisse). L'analyse de Vahlen peut-elle encore être valable si on ne lit pas in lapide ? Il fallait en tout cas tenir compte des objections à mon avis dignes de considération que Vahlen formule contre le fait, naturel et en apparence innocent, de rapporter hoc à lapide.

Fedeli admet la suppression (Richmond) des vers 125-126 de la seconde élégie(IB) inspirés par 65-66, et ponctue le célèbre morceau biographique ainsi:

$\begin{array}{cc}\text { Vmbria te notis antiqua Penatibus edit } & 121 \\ \text { (mentior ? An patriae tangitur ora tuae, } & 122 \\ \text { qua nebulosa cauo rorat Meuania campo } & 123 \\ \text { et lacus aestiuis intepet Vmber aquis ?) } & 124\end{array}$

\footnotetext{
plus creuses, ignorent Havet à leurs dépens. Comme souvent, Havet offre une explication de la faute trop complexe: la substitution de regna à regia - faute par anticipation de magna aura amené un remaniement métrique.

${ }^{30}$ Voir K. Brugmann, Die Syntax des einfachen Satzes im Indogermanischen, Berlin/ Leipzig 1925, 56.
} 
ossaque legisti non illa aetate legenda patris et in tenues cogeris ipse Lares.
127

128

Cette ponctuation, argue-t-il (354), a, sur celle qui circonscrit la parenthèse au v. 122, l'avantage de donner une fonction à la coordination (ossa)que (v. 127 ) et d'autoriser une interprétation propre ("être contigu ») et non figurée (" être mentionné ") de tangitur. Mais la parenthèse est trop longue pour ne pas obscurcir (ossa)que, dont la fonction (joindre edit et legisti) est assez claire dans la ponctuation suivante:

$$
\begin{aligned}
& \text { Vmbria te notis antiqua Penatibus edit } \\
& \text { (mentior? An patriae tangitur ora tuae ?), } \\
& \text { qua nebulosa cauo rorat Meuania campo } \\
& \text { et lacus aestiuis intepet Vmber aquis, } \\
& \text { ossaque legisti non illa aetate legenda. }
\end{aligned}
$$

De surcroît, la relative $q u a \ldots$ aquis se rapporte plus facilement et avec plus de pertinence au v. 121 qu'à la proposition An patriae tangitur ora. Fedeli ne se demande pas si et comment (indépendamment de la question de savoir si le sens obtenu est satisfaisant) le latin peut signifier " l'extrémité de ta patrie n'est-elle pas contiguë à la région où... ». Il attribue, sans donner de références ${ }^{31}$, à Markland, ainsi gratifié d'une construction pratiquement impossible, la ponctuation qu'il adopte, mais il ne dit pas que Markland change tangitur en tenditur, qui change la donne du point de vue de la syntaxe.

La ponctuation imprimée en III 67-69 est à rejeter absolument. Comme le commentaire (587) défend la bonne ponctuation, il doit s'agir d'une erreur. En XI 13, Fedeli lit Non minus immites habui, Cornelia, Parcas là où Heyworth a Num minus immites habuit Cornelia Parcas? La position de Fedeli se défend, mais il ne faut pas mettre entre virgules Cornelia, apposition au sujet: « quoique je fusse Cornelia » (« io, Cornelia » Fedeli 1300). Fedeli me paraît s'être laissé égarer par Heyworth, qui admet au v. 66 de la " reine des élégies " la conjecture de Peerlkamp, Vidimus et fratrem sellam geminasse curulem, I consule quo, fausto tempore, rapta soror, pour consule quo facto tempore rapta soror. Pour bien comprendre cette phrase, il suffit de ponctuer avant facto: " mon frère devenu consul, sa sœur (moi, Cornélie) pouvait mourir: le moment n'était pas inopportun ". À la différence de Heyworth, Fedeli connaît cette interprétation; il l'écarte au motif que sic (c'est-à-dire tempore) mori dicuntur, qui morte tempora iniqua et dura effugerunt (Hertzberg), mais Fedeli s'est laissé abuser par Hertzberg, car il est évident que tempore peut très bien signifier ici que, pour Cornélie, mourir

${ }^{31}$ Voir le Properce de Burman le Jeune, Utrecht 1780, 728. 
après avoir vu son frère devenir consul, ce n'était pas mourir trop tôt ou trop tard, mais au bon moment. Dans cette interprétation, tempore est une pointe beaucoup plus élégante et raffinée que l'épais fausto tempore.

\section{UN POINT D'ONOMASTIQUE ET UN POINT DE GRAMMAIRE}

II 51 Tempore quo sociis uenit Lycomedius armis atque Sabina feri contudit arma Tati.

Si les éditeurs de Properce fréquentaient la célèbre monographie Die Etrusker de Karl Ottfried Müller (seconde édition préparée et annotée par l'éminent étruscologue W. Deecke, Stuttgart 1877) et un ouvrage de Corssen plus sulfureux que Über Aussprache, je veux dire Ueber die Sprache der Etrusker, Leipzig, 1874-1875, maintiendraient-ils sans exprimer l'ombre d'un doute ou sans un examen plus approfondi la graphie Lycmon en I 29 (lygmon l'archétype selon Heyworth) et la leçon Lycomedius (licomodius F) ? Fedeli 207 et 209 n'affronte pas une difficulté que permet de saisir la discussion de Müller I 110-111 n. 125: comment expliquer Lycmon à côté de Luceres $^{32}$ v. 31 ? " Die Verse (...) nicht auseinander zu reissen sind, da der $L_{u c m o}{ }^{33}$ eben gegen Tatius kam, und diese beiden nebst Romulus eben die alten drei Tribus mit den Ihrigen gebildet haben sollen " (voir également p. 337-338 avec la note 18 de Deecke). Un fait qui corroborerait l'intention d'établir un lien entre Lucmo et Luceres, c'est que, chez Properce, la quantité $\mathrm{du}$ - $u$-dans Luceres est, comme dans Lucumo, brève, tandis qu'elle est longue dans le passage symétrique des Fastes d'Ovide III 132, chez qui je suppose que le rapport avec lux, lucis (-u-long) a prévalu ${ }^{34}$. Ciccarelli 487 approuve Fedeli 207, qui voit en Lycomedius la forme " romanizzata » de Lycmon: il semble curieux d'appeler "forme romanisée " un composé qui a un tel air

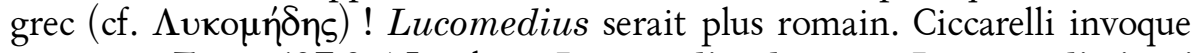
justement Festus 107,3-4 Lindsay, Lucomedi a duce suo Lucumo dicti qui postea Lucereses sunt appellati. Schulze Zur Geschichte lateinischer Eigennamen 92 rapproche Lucomedi, Lycomedius et Ratumedius (CIL V 4457, Brescia [Ratumedia !]). Dumézil ${ }^{35}$ explique Lycomedius et Lygmon (la forme transmise) par la volonté d'évoquer les guerriers-loups en rappelant le grec $\lambda$ úkos. Dans son édition de Festus, Leipzig 1839 120, Müller, au passage portant sur Lucomedi, a cette note: ex his intellegere mihi

${ }^{32}$ Étrusque lu Xre: cf. W. Schulze, Zur Geschichte lateinischer Eigennamen, Berlin 1933, $182,218,581$.

${ }_{33}$ "Lucmo, Scaliger, Turnebus », indique Smyth 1970.

${ }^{34}$ Voir ci-après n. 39. W. Corssen, Über die Sprache der Etrusker, Leipzig 1874, I, 251 égare son lecteur en ne présentant que la prosodie ovidienne, quand sa propre argumentation eût tiré meilleur profit de la prosodie propertienne.

${ }^{35}$ G. Dumézil, "Propertiana”, Latomus 10, 1951, $296-9$. 
videor, Graeculum aliquem vel Romanum immodice graecissantem Lycomidarum gentem, quae a Lycomo vel Lucomo nomen et originem ducebat, ex Attica Messeniave in Italiam transvexisse, ac Lucerum stirpem ex ea derivasse. Après avoir assimilé Lucumo (Lucumus chez Festus !) à ${ }^{*}$ Lykomos $^{36}$ (opposer Lycmon chez Properce), ancêtre des Lycomides (Lykom-idai), grande famille chargée du culte des Mystères à $\mathrm{Phlya}^{37}$ et à laquelle appartient Thémistocle, on aurait ramené Lucumones

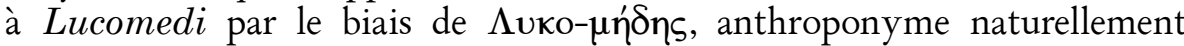
affectionné des Lycomides. Un érudit ancien expliqua-t-il par $\Lambda v \kappa o \mu \eta \dot{\delta} \eta \bar{s}$ l'étrusque Luc-u-mo, dont la formation (Corssen Die Sprache der Etrusker I 251-252, II 253) n'apparaissait pas ? Il se peut aussi que Lykos, guerrier ${ }^{38}$ éponyme des Lycomides réputé avoir fondé le culte d'Apollon Lycien compris comme "Apollon au loup $»^{39}$ - et gardien du droit et de la justice ${ }^{40}$, ait contribué à établir un lien entre les Lucumones, ces chefs étrusques, et les Lycomides et que ce même lien explique, dans le sens voulu par Dumézil ${ }^{41}$, la graphie latine avec $y$. On n'était peut-être pas non plus mécontent de trouver un lien entre les Étrusques et la Lycie, réputée, selon une version ${ }^{42}$, fondée par notre Lykos et point si éloignée de la Lydie.

Il y a toutefois chez Properce, dans le passage même de Lycmon à sa prétendue romanisation Lycomedius, un sujet d'étonnement. Lucumonius pour Lycomedius est une correction de Müller 1877110 n. $125^{43}$, adoptée par

${ }^{36}$ Voir H. Usener, Götternamen: Versuch einer Lehre von der religiösen Begriffsbildung, Frankfurt $1948^{3}, 213$.

${ }^{37}$ Voir encore le chapitre que leur consacre J. Toepffer, Attische Genealogie, Berlin 1889, 208-25.

${ }^{38}$ Toepffer 219.

${ }^{39}$ Pausanias I 19,3 avec le commentaire de Frazer Londres 1898 II 195; O. Gruppe, Griechische Mythologie und Religionsgeschichte, München 1909, 1236-7; U. von Wilamowitz, Die Glaube der Hellenen, Darmstadt 195933, I 143-144; W. Burkert, Homo necans, Berkeley/ Los Angeles 1983, 121. L'étymologie qui veut rattacher l'un et l'autre mot à la racine signifiant l'éclat (cf. Corssen, Über Aussprache, I 367; Usener, Götternamen, 198 ss.) réunirait « Ly-

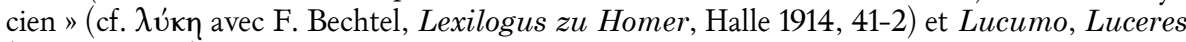
(chez Properce). Avec le "gouna " on obtient Lauc-, par exemple dans Laucumnia, que nous évoquons plus bas, mais aussi, avec un changement de degré, lux avec $u$ long en opposition à lucerna avec $u$ bref. M. de Vaan, Etymological Dictionary of Latin and the other Italic Languages, Leiden/Boston 2008, 356 dit à propos de lucerna que " its short - $u$ - is unexplained ". Ce n'est qu'un exemple de changement de degré parmi d'autres (cf. K. Brugmann Grundriss der vergleichenden Grammatik der indogermanischen Sprachen, Zweite Bearbeitung, I, Strasbourg 1897, 487).

${ }^{40}$ Selon Usener Götternamen 213-216, Lykos est le dieu lumineux qui incarne la justice et protège l'institution judiciaire et notamment les héliastes. D'après l'illustre érudit, l'étymologie populaire a dégradé en "loup » ce lumineux gardien du droit.

${ }^{41}$ F. Dümmler, "Einige eleusinische Denkmäler”, Kleine Schriften III, Leipzig 1901, 37 évoque les " wilden Vorväter » des Lycomides.

${ }_{42}$ Voir Hérodote I 173,3 avec la note de D. Asheri, A Commentary on Herodotus Books $I-I V$, edited by Oswyn Murray and Alfonso Moreno, Oxford 2007, 195.

${ }^{43}$ Il n'en est pas le premier inventeur: «Lucumonius Annius, Scaliger, Turnebus », indique 
Corssen Die Sprache der Etrusker I 251. Lucumonius ${ }^{44}$ n'est pas moins un hapax que Lycomedius. Quel serait le sens de Lucumonius ? Singulier mis pour le pluriel et désignant un ensemble d'individus (sur cet ensemble, voir Deecke chez Müller I 466) ou singulier désignant un individu nommé Lucmo? Dans ce dernier cas, Lucumonius ne saurait être purement et simplement l'équivalent de Lucmo, mais il s'agirait d'une périphrase, "le Lucumonien " $=L u c m o o^{45}$. Selon Müller I 338 n. 21, si Properce avait vu en Lucmo un nom propre et non un appellatif ${ }^{46}$, le passage de Lucmo à Lucumonius serait «très surprenant». Donc Lucmo = Lucumo serait ici « eine allgemeinere Bezeichnung vornehmer Tusker ». Je croirais plutôt que Lucmo, opposé à Tatius en I 29 (cf. aussi II 51), était pour Properce un anthroponyme et je gagerais que pour lui Lucumonius (si c'est bien ce que le poète avait écrit) signifiait « l'éponyme des Luceres lui-même appelé Lucmo ": Luceres/Lucerenses sont des pluralia tantum et Properce ne pouvait pas désigner Lucmo par le singulier Lucer/ Lucerensis. Lucumonius avait en plus l'avantage d'indiquer l'anthroponyme. Observer la cohérence de l'ensemble Luceres-Lucmo-Lucumonius, où Lucmo (cf. ser-mo, pul-mo) n'est autre que la réduction de $L_{u c u m o}{ }^{47}$ et opposer Luceres-Lycmon-Lycomedius, où Lycmon hellénise Lucmo en paraissant se rapprocher d'une formation telle que Ac-mon ou Id-mon. Lycmon n'est pas attesté en grec, où $\Lambda$ okó $\mu \omega v$ ou $\Lambda$ ovkoú $\mu \omega v$ rend, d'une manière attendue ${ }^{48}$, Lucumo (mot anapestique). Je conclus cette analyse condensée en remarquant que si, en l'état de nos connaissances, il peut paraître plus prudent de lire Lycmon et Lycomedius, il n'en reste pas moins qu'il y a lieu d'envisager Lucmo - virtuellement condamné à devenir Lyc/gmon à côté de Haemon, Lyc/ gurgus, Lygdamus - et peut-être Lucomedius - à moins que Müller n'ait raison de lire Lucumonius, qu'un diorthote aurait remplacé par une forme plus familière, qui lui rappelait les gentilices en -edius que mentionne Schulze 92, ou plus proche de Lucomedi. De Luc[u]mo / Lucomedius (désignant le même individu) rapprocher peut-être Ratumenna (cf. ratumsna, raAumnsnal) / Ratumedius (Ratumedia), que je tire de Schulze 92.

Smyth, dans une note incomplète qui paraît tirée de l'édition de Valpy, Londres 1822636 , lequel renvoie à Scioppius.

${ }^{44}$ Rapprocher Laucumnia Felicitas (CIL XI 1788 Volterra; Schulze 179).

${ }^{45}$ Fedeli 207 explique que celui que Properce est censé appeler Lycmon " è il re etrusco che nella guerra fra Romani e Sabini, guidati da Tito Tazio, si schierò dalla parte di Romolo ", mais le passage de Varron (" ap. Serv. ad Verg. Aen. 5,560») qu'il allègue ne montre rien de tel: voir les textes que cite, entre autres érudits, Gilbert, Geschichte und Topographie, II, Leipzig 1885, 64 n. 1.

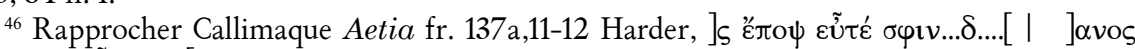

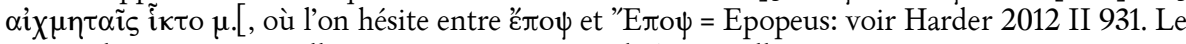
rapprochement entre Callimaque et Properce est dû à A. Hollis.

${ }^{47}$ Comparer Schulze 179: «lauXumsnei lauXmsnei [CIE] 2387 sq. (Clusium) gewähren die schönste Parallele für Arcumenna: arcmsnei, Ratumenna: ratumnsna ».

${ }^{48}$ Corssen, Die Sprache der Etrusker I, 251. 
XI 5-6 Te licet orantem fuscae deus audiat aulae, nempe tuas lacrimas litora surda bibent.

La correction limina (Fontein) vient immédiatement à l'esprit (cf. 2 panditur ad nullas ianua nigra preces), mais ce n'est pas le problème posé par litora qui m’intéresse. "Cornelia, écrit Fedeli 1292, opera un sottile 'distinguo': ammetiam pure (licet) che le preghiere giungano alle orecchie (audiat) di Plutone; in ogni caso le lacrime sono destinate a venire assorbite dall'Acheronte ». Fedeli n'explique pas le sens qu'il donne à nempe, « in ogni caso ». Pourtant, cet emploi de nempe appelait une remarque: comparer Hand, Tursellinus IV 158 (il a bien entendu le passage); Kühner-Stegmann I 809; OLD s. v. nempe 1d; Hutchinson 2006 233. Nempe n'est en réalité ni concessif ni adversatif (" yet certainly », $O L D$ ): « en admettant qu'il entende, eh bien il n'écoutera pas ", ut audiat, nempe tamen (Lucrèce II 908; Ovide Pont. IV 3,38; etc.) non exaudiet. Cette explication frappe de caducité à la fois la permutation par Goold des pentamètres 2 et 6 (Fedeli ne la mentionne pas) et la remarque de Heyworth (Cynthia 504), " the deafness of the shore does not clearly trump the potential willingness of the deus to lend an ear, so nempe asserts a logical relationship that is not entirely firm ".

\section{CORRUPTIONS VERBALES ET CONJECTURES NON SATISFAISANTES}

Le maintien d'un nombre à mon avis trop élevé de leçons fautives est peutêtre le défaut le plus sérieux de ce commentaire, soit que les auteurs passent sous silence les difficultés soit qu'ils les apprécient mal. Dans ce dernier cas, il leur arrive d'opter pour des conjectures problématiques. Procédons, pour le choix d'observations en général brèves qui suit, élégie par élégie.

I A. L'hexamètre hinc Tities Ramnesque uiri Luceresque Soloni $\left(31^{49}\right)$ présente un déséquilibre suspect entre uiri et $\operatorname{Soloni}(=$ Solonii, originaires de la cité de Solonium, dont, selon Denys d'Halicarnasse, provient Lucumo ${ }^{50}$ ), présumé être la variante la plus autorisée (v. 1. coloni) et " an accurate piece of arcane knowledge... most unlikely to hare arisen from intrusion, never mind corruption " (Heyworth Cynthia 418). Viri donne l'impression d'un bouche-trou (amélioré par le rendu " héroïques » de Heyworth); feri (Palmer) maintient le déséquilibre et appellerait plutôt le symétrique seueri (Housman). Le déséquilibre incriminé disparaîtrait si on lisait hinc Tities Ramnesque tribus Luceresque Soloni, " voilà les origines (très modestes)

${ }^{49}$ On me permettra de m'étonner de l'absence d'un renvoi, à propos de ce vers, à Schulze, Zur Geschichte lateinischer Eigennamen, 218, et de l'ignorance où les auteurs paraissent être de ce livre fondamental.

${ }^{50}$ Voir Deecke dans sa réédition de l'ouvrage de K. O. Müller, Die Etrusker I, Stuttgart 1877, 110 n. 124 et R. E. A. Palmer, The Archaic Community of the Romans, Cambridge 1970, 144 . 
des tribus que formèrent les Tities, les Ramnes et les Luceres soloniens" (Tite-Live X 6,7 ut tres antiquae tribus, Ramnes Titienses Luceres) ou, mieux encore, hinc Tities Ramnesque equites Luceresque Soloni et peutêtre même hinc Tities, Ramnes celeres Luceresque Soloni: celeres est le terme technique archaïque pour dire equites (Tite-Live I 15,8); la mécoupure Ramnesce *leres devait amener un remaniement tel que Ramnesque uiri. On sait que Tities, Ramnes et Luceres forment trois centuriae equitum (Tite-Live I 13,8). Pour equites ou celeres particulièrement rapproché de Ramnes (mais valant pour les trois éléments), voir Horace Art Poétique 342 celsi (...) Ramnes, où celsi est "vox propria für den stolzen Reitersmann " (Kiessling-Heinze $)^{51}$. La défense de uera fuere au v. 52, aut si Pergameae sero rata carmina uatis | longaeuum ad Priami uera fuere caput, est fourvoyée: le sens que cherche Fedeli en forçant ad serait donné par la conjecture lata fuere de Heyworth, si ce n'était un solécisme (voir Madvig Opuscula academica 1887, 576-583) : transiluere est une possibilité. Les passages qu'allègue Heyworth 2007421 sont différents de lata fuere soit du point de vue du temps et/ou du mode soit du point de vue du sens et l'un d'eux est à lire autrement : I 4,9, fuerit collata ; 15,35, mentita fuisses ; II 26,3, fueras mentita ; 28,21, fuerat deuota ; 31,2, aperta fuit (cf. Madvig 580) ; III 10,29, fuerit... exacta ; 23,11, forsitan... fuerunt mandata (v. l. fuerint) ; IV 7,8, lateri uestis adusta fuit (tournure possessive !).” Au v. 54, Ilia tellus $\mid$ uiuet et huic cineri Iuppiter arma dabit, la conjecture arua (Garrod) mérite considération. Nous avons évoqué plus haut le v. 66.

I B. Au v. 71, le sens requiert Fuge discere fata, Properti ! (texte de Heyworth), non Caue (uage mss.) dicere fata. Le texte corrigé Accersis lacrimas cantans; auersus Apollo (73) ne convient pas puisqu'Apollon conseille à Properce d'être poète. Le texte adopté par Heyworth, Accersis lacrimas: auersus cantat Apollo, se tient. Au v. 94, heu sibi prolapso non bene cauit equo, Heyworth a raison de permuter bene et sibi en ponctuant heu bene prolapso, non sibi, cauit equo. L'adjectif uerus au v. 107, Aspicienda uia est caeli uerusque per astra | trames, est faux: caeli a peut-être absorbé la première syllabe de limus " oblique ». Lire Aspicienda plaga est caeli ? L'euphémisme dilige dans Victor Oiliade, rape nunc et dilige uatem (117), est bizarre: pollue, comprime conviendraient. Dans le distique biographique 131-132, Mox ubi bulla rudi dimissa est aurea collo, I matris et ante deos libera sumpta toga, le syntagme matris ante deos semble faire difficulté, même si la bulla était abandonnée aux Lares, ce qui

${ }^{51}$ Si l'on voulait entendre " les rapides Ramnes ", on pourrait signaler que Corssen, Über Aussprache, II 85, rattache Ramnes à la famille de rap-ere, fait valoir leur réputation de " racaille prédatrice » ou d'enleveurs de jeunes filles mais suggère que Ramnes exprimait originellement l'idée de célérité. 
suggère ante Lares. Une autre possibilité est ante oculos. Au vers suivant, tum tibi pauca suo de carmine dictat Apollo, il faut peut-être pectore à la place de carmine. Fedeli lit Et bene cum fixum mento decusseris uncum, †il erit hoc: rostro te premet ansa suo (141-142) et entend (384): « e per quanto cercherai di strappar via il gancio infisso al mento, non ci sarà niente da fare: la sua ansa farà forza su di te (= sul tuo mento) con la sua punta ". Explication à mon avis peu convaincante de rostro... ansa suo. La tradition donne premit ou premat, ausa, nostro et hésite entre suo et tuo; je suggère rostro te reget ipsa (ipsa Heyworth; peut-être illa) tuo, " elle saura bien te mener par le bout du nez ". Rostrum en ce sens (OLD s. v. 1c) est familier, ton qui n'est pas étranger à la poésie de Properce.

II. Ciccarelli ne prend la mesure des difficultés ni du premier vers, Quid mirare meas tot in uno corpore formas? ni du second, accipe Vertumni signa paterna dei, où, sinon les trois derniers, du moins les deux derniers mots semblent devoir être mis inter cruces. Il me paraît évident que Properce avait, comme le veut une conjecture remontant à l'humanisme, formulé ainsi le début du poème: Qui mirare meas tot in uno corpore formas, accipe... Ciccarelli cite elle-même le mouvement analogue de l'incipit de I 21: on pourrait aussi mentionner celui de l'épigramme 22. La suite, mystérieuse tant qu'on n'aura pas de témoins plus fiables que ceux de notre tradition, pourrait bien être non le verbiage inane signa paterna dei, mais fata canente deo ${ }^{52}$, " écoute le destin de Vertumne, chanté par le dieu lui-même ", conjecture que m'inspire la correction brillante mais non totalement satisfaisante accipe Vertumni signa fatente deo de Shackleton Bailey (cf. 20 de se narranti tu modo crede deo). Car ce dieu a bien eu un destin et c'est aussi lui qu'il raconte, tout en formant des vœux pour qu'il se perpétue indéfiniment (5556). Nous savons, nous autres, que ce destin fut moins flatteur que ne le voulait ou ne le savait Properce: l'étrusque Voltumna ${ }^{53}$ était une divinité autrement deo.

${ }^{52}$ Comparer, pour la forme, Ovide (?) Hér. XXI 232 quaeritur a Delphis fata canente

${ }^{33}$ Ciccarelli cite la littérature récente. Noter que Corssen, Die Sprache der Etrusker, II 107, distingue encore Vol-t-u-mna de Vor-t-u-mnus et en fait la "'schützende' Bundesgöttin " des Étrusques, rapprochant pour l'étymologie Var-una-s, "schützender, deckender ». Pour Schulze, Zur Geschichte lateinischer Eigennamen, 252, la déesse Voltumna " ist nichts anderes als die Familiengottheit der ultimni » et Vertumnus, qu'il distingue aussi de Voltumna, lui paraît aussi être une "divinité familiale " (Wissowa, article "Vertumnus » du lexique de Roscher VI 219, attribue à tort à Schulze l'idée que les deux théonymes n'en font qu'un: Schulze veut seulement dire qu'il s'agit pour lui de deux "divinités familiales "). Cette féminisation n'eût pas déplu au dieu qui déclare indue me Cois, fiam non dura puella (clin d'œil du poète à sa dura puella I 17,16, elle aussi vêtue de Coa, II 1,5). Pour ma part, si j'osais, je suggérerais un lien de parenté entre uol-tu-s = uultus et Voltumna, "celui (celle) qui voit », de la racine *wel, sur laquelle on verra, particulièrement à propos de sa productivité théonymique, M. L. West, Indo-European Myth and Poetry, Oxford 2007, 146-7. Pour la formation vol-t-à partir d'un élargissement de la racine, rapprocher par exemple anglo-saxon vlitan " voir " et peut-être 
plus importante en Étrurie que Vortumnus/Vertumnus à Rome. Signa se sera substitué à fata à cause des statues de Vertumnus que le dieu lui-même évoque. Une fois cette faute advenue et deo passant à dei sous l'influence de Vertumni, il devenait nécessaire de retoucher canente et, si creux soitil, paterna aura semblé, dans l'urgence, suffire. Rapprocher l'excellente émendation de J. B. Hall canentum pour le fautif parentum chez Stace Ach. 12. Aux v. 11-12, Seu, quia uertentis fructum praecerpimus anni, I Vertumni rursus credis id esse sacrum, l'adverbe rursus, "d'altra parte ", n'a pas de justification véritable et le id conjectural (credis id Postgate pour credidit des mss. autorisés) paraît gauche: Vertumno fructus creditur esse sacer (Ayrmann) exprime convenablement le sens requis. Ciccarelli ne s'interroge pas sur l'introduction chez Properce du peu poétique $i d^{54}$, qui au contraire enthousiasme la commentatrice: " il deittico $i d$, infatti, crea un rapporto di consequenzialità ( ?) tra la causa espressa nel verso precedente e il culto di Vertumno ». Les cerises mentionnées v. 15 sont douces, hic dulces cerasos, hic autumnalia pruna | cernis et aestiuo mora rubere die, mais autumnalia et aestiuo suggèrent uernos. Vertumnus semble une explication insérée au v. 35, Est etiam (mea et Postgate 1897, mihi et Hanslik) aurigae species Vertumnus (cum uerbere Postgate 1894) et eius | traicit alterno qui leue pondus (corpus Passerat) equo. Scaliger, dans sa note à Manilius V 85 (p. 391 de la Plantiniana de 1600), Corssen Über Aussprache II 171 et Postgate Select Elegies of Propertius 1881202 postulent un nom commun uertumnus. Mais, tandis que Scaliger rapporte uertumnus à un " genre d'auriges " qui passe d'un quadrige à l'autre, Corssen rapporte uertumnus au desultor qui passe d'un cheval à l'autre. Ni Corssen ni Postgate 1881 ne s'expliquent davantage: entendent-ils « il (Vertumnus) est aussi uertumnus, un genre d'aurige et d'homme qui fait passer légèrement son corps d'un cheval à l'autre "? Ciccarelli, dont les explications ne sont pas très claires, entend ainsi sauf qu'elle lit Vertumnus. Le passage a besoin d'un examen approfondi: ce que les commentateurs offrent est précisément "désultoire ", désinvolte ${ }^{55}$.

gothique wulthus " gloire " si l'on en croit Walde cité dans Walde-Hoffmann, Lateinisches etymologisches Wörterbuch ${ }^{3}$, II 831 à l'article voltus. Pour le sens actif du suffixe participial, voir Corssen, Über Aussprache, II 170 ss. Si l'on accepte l'équation Voltumna = Vortumnus, les Romains se sont - bien naturellement - mépris sur l'étymologie du théonyme; il resterait pourtant peut-être un écho, bien lointain, certes, de l'étymologie proposée dans Romanum satis est posse uidere forum (6).

${ }^{54}$ Unique occurrence, très gauche et certainement fautive, chez Properce en I 20,2, où l'on verra la note de mon commentaire (à paraître).

${ }^{55}$ La page de L. Friedländer dans Le Culte chez les Romains. Les jeux, trad. Brissaud, II Paris 1890, 299 et l'article desultor de Saglio dans le Daremberg-Saglio II 111A-113A sont insuffisants. Scaliger et Friedländer citent le passage de Firmicus Maternus (VIII 6) où il est question de qui saltu quadrigas transeat. Selon Franz Skutsch, Kleine Schriften, Leipzig 1914, 419, quadrigas n'est pas nécessairement un contresens (hypothèse de Scaliger) sur quadrupedum 
Un examen plus critique doit aussi être accordé aux v. 37-40, ainsi imprimés par Ciccarelli:

Suppetat hic, pisces calamo praedabor et ibo mundus demissis institor in tunicis.

Pastor me ad baculum possum curuare uel idem sirpiculis medio puluere ferre rosam.

Suppetat hic paraît un aménagement très insuffisant de la leçon traditionnelle fautive suppet(/r)at hoc, pour quoi Heyworth adopte l'ingénieux sub petaso (Alton). Pastor me (Ayrmann) pour pastorem et curuare (cj. ancienne anonyme) pour curare ne semblent pas non plus heureux; le contexte requiert pastor ouem (ainsi déjà Huleatt, avec ouem singulier collectif, comme plus loin rosam) et un verbe qui pourrait être sinon curare (ad baculum = baculo innixus, Ovide Mét. VIII 218), du moins numerare (cf. II 1,44 enumerat miles uulnera, pastor oues) ou seruare. Pour le dernier pentamètre, Ciccarelli 476 juxtapose des conjectures très libres (ainsi l'obscur et bizarre sirpiculis media plebe referre rosam) qu'elle agrémente de remarques non appréciatives. Peut-être suffit-il de corriger medio en moto: le commis s'efforce d'être rapide, image de célérité opposée à celle du berger qui se tient sur son bâton. La pertinence de probata dans hortorum in manibus dona probata meis (42) n'apparaît guère: parata introduirait une expression bien attestée dans le registre de l'offrande. Dans le distique 45-46 nec flos ullus hiat pratis, quin ille decenter I impositus fronti langueat ante meae, l'adverbe ante est vide de sens s'il ne devient préposition, frontem ante meam (Fea). Il y a peut-être un effet de surprise et un trait d'humour dans langueat; on aurait attendu luceat: cf. la traduction de La Roche-Aymon (Paris 1885), En nul temps dans les prés ne s'élève une fleur $\mid$ Qui ne vienne à mon front étaler sa couleur, rendu qui évoque I 2,9 Aspice quos summittat humus †formosa† colores. Au v. 60, Ciccarelli préfère le bouche-trou transmis grata (pauper in urbe deus) au joli pauper paupere in urbe deus de Heyworth: je parie qu'elle a tort. Nous avons traité plus haut le problème posé par la leçon Lycomedius (51).

de Manilius V 86 mais peut provenir de V 73 stare leui curru moderantem quattuor ora (auquel cas il y a quand même contresens!). Pour ma part, je me demande si Maternus ne lisait pas chez Manilius nec non alterno desultor sidere dorso | quadriiugum (gén. pl., relevé chez Ennode par Neue-Wagener IV 296), ce qui expliquerait son erreur: il s'agit de passer d'un cheval à l'autre du quadrige, non d'un quadrige à l'autre. Le passage de Firmicus n'apparaît pas dans la communication de J.-P. Thuillier "Les desultores de l'Italie antique", CRAI 133, 1989, 33-53. Quant au passage de Properce, Thuillier se contente de le citer (49) en remarquant que "le poète, s'il n'emploie pas le mot lui-même [desultor], compare cependant sans ambiguïé si l'on peut dire - le dieu Vertumnus, toujours changeant, à un voltigeur sautant avec légèreté d'un cheval sur l'autre». 
III. Les croix de la désespérance devraient inclure le substantif noctes(11), quoi qu'en ait Dimundo, qui ne rend pas justice à la restitution de Watt et pacta haec foedera nobis. Si elle discute noxia (d'une façon à mon avis peu satisfaisante), Dimundo ne dit pas un mot sur l'homéotéleute pendent mea noxia uota (17)et une partie des mérites de la correction de Guyet mea pendent anxia uota lui échappe. Je trouve tordues les explications qu'on va chercher pour maintenir obliquo contre obliquum dans dignior obliquo funem qui torqueat Ocno (21). Au v. 34, Dimundo paraît avoir raison d'adopter le verbe suo (Rossberg) en lieu et place de suos (sc. gladios, corrigé en chlamydas par Barber) mais tort de conserver uellera secta, à quoi Heinsius substitue uellera lecta: et Tyria in chlamydas uellera lecta suo. Heyworth élimine le distique 37-38, cogor et e tabula pictos ediscere mundos, I qualis et haec docti sit positura dei et, si tel est le texte original de ce distique, on ne peut qu'approuver cette athétèse. Mais le distique est plus vraisemblablement corrompu: pictum condiscere mundum et Arctoi (Arctoi déjà Hetzel) sit positura poli ont l'air plausibles. En maintenant essem contre issem (Heinsius) dans essem militiae sarcina fida tuae, Dimundo néglige à la fois la phraséologie latine et la "vraisemblance paléographique " à laquelle elle est par ailleurs attachée, à contresens parfois. Pater peut-il subsister tout seul dans le texte conjectural qu'elle adopte nec me tardarent Scythiae iuga, cum Pater altas | astricto in glaciem frigore uertit aquas (47-48) ? On voudrait que Dimundo cite en toutes lettres un parallèle digne de ce nom. Le redondant astricto corrige *affricus, qui a paru, je suppose, recouvrir astrictus mais semble recouvrir plutôt astricus, adjectif rare attesté dans les Satires Ménippées de Varron et chez Jules Valère. Properce a-t-il pu dire pater astricus ou doit-on astricus à un docte ancien ou médiéval ? Le poète aura plus vraisemblablement dit pater aetheris ou aeris (Rossberg). Dimundo lit uertit (Morgan), mais son commentaire défend la leçon transmise nectit: si, de fait, le texte transmis avait été uertit, j’aurais trouvé intéressante la conjecture nectit. In glaciem uertit est évidemment plus simple, mais, avec nectit, la locution in glaciem, résultative, est idiomatique. Aperit clausos una puella lares (54) est en faveur de limina (Heinsius) surda (55), non de omnia surda que défend Dimundo à coup de parallèles de pertinence limitée. Le v. 56 est relatif à une petite chienne nommée Craugis et illa tui partem uindicat una toro est une faute manifeste pour illa tuam partem uindicat una tori, correction de Guyet ou, mieux encore, illa tori partem uindicat una tuam (Heyworth). Au v. 64, avec picta, Palmer enlève une glose insérée dans le texte, raptaue odorato carbasa lina duci, maintenu tel quel par Dimundo: ce qui avant tout dénonce la glose, ce n'est pas le fait que carbasa soit mis pour carbasea; c'est la tautologie carbasa lina, que le commentaire occulte. 
IV. Nous avons discuté plus haut la ponctuation du v. 13. Au v. 7, qui suit le vers 14 dans le texte de Fedeli et qui est transmis ainsi, Hunc Tatius fontem uallo praecingit acerno, il est difficile de ne pas voir en fontem une faute par persévérance due à fonte (v. 14). Fedeli accepte la substitution de laticem (Barber) au même fontem aux v. 15-16, Hinc Tarpeia deae fontem libauit: at illi | urgebat medium fictilis urna caput. Fedeli rend avec Camps le parfait par "went to draw ", mais libare signifie, si je ne m'abuse, " offrir en libation ", non " puiser ": tel est aussi le cas en III 17,38, libatum fundens in tua sacra merum, "versant en libation le vin pur en ton honneur ", passage que cite Fedeli pour illustrer le sens de " prendre de l'eau ". Je doute que le parfait puisse dans ce contexte signifier libatum iit, le plus-que-parfait libarat (Fontein) libatum ierat, et je crois plus volontiers que libabat (Hutchinson) peut vouloir dire libatum ibat ou libaturus erat. Hutchinson y voit un imparfait de répétition; c'est l'aspect de l'action qui m'intéresse, dans la mesure où il rend possible l'idée que le moment en vue est celui où Tarpeia emporte l'eau qu'elle vient de puiser afin d'en faire libation. Fedeli s'efforce de justifier medium caput: je crois qu'il faut summum caput et j'en veux pour preuve le passage qu'il cite lui-même (629), ponitur e summa fictilis urna coma (Ovide Fastes III 14). Je regrette que Fedeli suive Heyworth en écartant tum (Rossberg) pour tu (v. 48), mais c'est surtout le problème du v. 50 qui m'intéresse: Lubrica tota uia est et perfida: quippe latentes | fallaci celat limite semper aquas. Semper cache le sujet de celat: caespes (Palmer) ou peut-être muscus. Au v. 61, les éditeurs paraissent insensibles à la difficulté de Adde, Hymenaee, modos ! Pourtant Ede (Guyet) est une conjecture évidente. Bracchia (v. 28) a peut-être amené une faute par persévérance au v. 67, Dixit et incerto permisit bracchia somno: Fedeli brode sur l'emploi de bracchia ici, mais Properce a dû écrire lumina ou pectora (Markland), sinon corpora. Tels que Heyworth et Fedeli les impriment, les v. 73-78 ne peuvent être attribués à Properce, même si Fedeli substitue deliciis à diuitiis au v. 76, cum pagana madent fercula diuitiis. Il faut peut-être natant, qui est propre à indiquer l'abondance, à la place de madent. Immundos (v. 78) est particulièrement ridicule dans le distique cumque super raros faeni flammantis aceruos | traicit immundos ebria turba pedes. La solution la plus simple est immunis " indemnes ". Aux v. 73-74, le meilleur moyen de mettre en relation festus et dies est de substituer qui (Phillimore) à hic: Vrbi festus erat (dixere Parilia patres), | qui primus coepit moenibus esse, dies. Vrbi (urui Richmond) n'est guère moins suspect au début du v. 73 que urbe à la fin du v. 75, annua pastorum conuiuia, lusus in urbe (herba Fontein, umbra Housman). Le rejet de la correction dapibus (Jacob) pour dubius v. 83 entraîne la malheureuse mise inter cruces de festoque remissus: pourtant, Mons erat ascensus, dapibus festoque remissus semble irréprochable. Fedeli aurait mieux fait ici de s'inspirer de Goold que de Heyworth. Au v. 85, Omnia praebebant somnos, que Fedeli maintient, 
est tout à fait incongru: Heyworth opte pour omnia praebebat somnus (Markland), mais omnia carpebant somnos (Lütjohann) quadre mieux avec le contexte. Comme Heyworth, Fedeli accepte la transposition, après le v. 86, des v. 17-18, Et satis una malae potuit mors esse puellae|quae uoluit flammas fallere, Vesta, tuas? La question rhétorique est, en l'état, formulée avec une improbable maladresse; s'il faut garder le distique (et je crois que Hutchinson a tort de l'athétiser), il convient vraisemblablement de lire Nec satis (Postgate) et de supprimer le point d'interrogation. Properce n'a pu écrire le distique final que lui prête Fedeli, $A$ duce Tarpeium mons est cognomen adeptus. $\mid$ O uigil, iniustae praemia sortis habes. Au moins possible et sensé serait $A$ nece Tarpeiae (Bassanus) mons est cognomen adeptus. I Haec o uix iuste praemia fraudis (Damsté) habes.

V. Dans et tua, quod non uis, sentiat umbra sitim (2), Dimundo aurait dû étendre les cruces à quod non uis et non seulement à non uis. Si Heyworth n'a pas rencontré le vrai avec son perpetuam, perdu après tua et remplacé par un bouche-trou particulièrement malheureux - l'objection de Dimundo, selon qui perpetuam n'a pas de plausibilité paléographique, porte à faux -, je risque Tantaleam. Dimundo admet cineri ablatif ${ }^{56}$ dans nec sedeant cineri Manes (3), " may your ghost not settle in the ashes " (Heyworth). Mais que cineri soit ablatif ou datif, la diction est obscure et suspecte. Rothstein cite comme inspiré du passage de Properce CLE 197,2 adsint quieti cineribus Manes tuis (cf. Shackleton Bailey 1956 240), ce qui suggère chez Properce lui-même adsint nec cineri Manes (cf. 50 nauta nec...; I 229 unica nec desit...; III 2235 cornua nec ualuit; IV 856 spectaclum capta nec minus urbe fuit; IV 11,94 caelibis ad curas nec uacet ulla uia). La restitution fautive de l'ordre habituel nec adsint aura entraîné un remaniement.

Dimundo défend le futur poterit dans Illa uelit, poterit magnes non ducere ferrum (v. 9) en minorant le problème posé par le fait que les verbes des autres phrases sont au passé: le maintien du futur est cher payé quand poterat (Fontein) s'offre de lui-même. Heyworth lui-même conserve poterit mais traduit " would ", c'est-à-dire poterat. Il est vrai que, dans le distique Quippe et, Collinas ad fossam mouerit herbas, I stantia currenti diluerentur aqua (11-12), Dimundo met mouerit sur le même plan que uelit et poterit, difficiles, dit-elle, à concilier avec « l'idea che la lena sia già morta "; pourtant, mouerit n'est pas un potentiel et, si on lit poterat, le présent uelit équivaut à uellet ou uoluerit, conformément à une liberté dans la concordance des temps qui s'explique par la commodité métrique et que Dimundo 734 illustre. Elle veut que ad fossam mouerit herbas signifie " mescolare nel

${ }^{56}$ Forme (ablatif instrumental -id) non encore totalement éliminée par la forme concurrente en $-e$ (K. Brugmann, Grundriss der vergleichenden Grammatik, II 2, Strasbourg 1890, $593-4)$. 
calderone presso una fossa ": c'est à mon avis tout à fait improbable. Si ces mots sont correctement transmis, ils doivent signifier " should she move herbs gathered at the Colline gate to a ditch " (Heyworth). Mais mouerit suggère presque irrésistiblement umbras (Heinsius), " évoquer les morts au bord du fossé »: les commentateurs signalent que les Vestales impures étaient enterrées vivantes dans le Campus Sceleratus derrière la Porte Colline, et c'est avec plus d'à-propos qu'il ne croyait que Rothstein comparait Horace Sat. I 30,28-29 cruor in fossam confusus, ut inde (" par ce moyen ») । Manis elicerent. Le participe substantivé stantia, qui, selon l'explication préférée par Dimundo, désignerait des roches, fait difficulté. S'il s'agit bien de pierres liquéfiées (car l'explication de stantia par "standing crops " produit un quasi non-sens), il faut un substantif adéquat pour les désigner et, s'il s'agit bien d'évoquer les morts dans la proposition précédente, on ne peut plus faire de cette proposition la protase de l'apodose dont le verbe est diluerentur, car l'évocation des morts et la liquéfaction des pierres n'ont pas de rapport entre elles. On serait donc amené à coordonner les deux propositions: Quippe et Collinas ad fossam mouerit umbras | saxaque currenti diluerentur aqua: " c'est qu'aussi ${ }^{57}$ elle pouvait évoquer les Ombres de la Porte Colline au bord du fossé et dissoudre les pierres en eau courante ". J'emprunte saxaque à Heinsius. Pour le subjonctif imparfait hors du système conditionnel au sens du potentiel du passé, comparer cogeret $\mathrm{v} .8^{58}$; pour le subjonctif parfait dans le même sens, voir Hofmann-Szantyr 334 b, non sans les réflexions pénétrantes de Delbrück Vergleichende Syntax II Strasbourg 1897 389-390.

Les cruces des v. 19-20 auraient dû inclure Exorabat opus uerbis et le double génitif Eurypylique... Coae textura Mineruae (23) n'aurait pas dû être maintenu au détriment de la correction d'Heinsius Eurypylis. Les secta(que) ab Attalicis putria signa toris (24), " crumbling figures cut from Attalus' <golden> bed-covers » (Heyworth), provoquent la stupéfaction: il doit s'agir de broderie, prétendument inventée par Attale de Pergame, faite au moyen de fil d'or et l'on attendrait à peu près textaque in Attalicis aurea signa toris ${ }^{59}$, "les figures tissées en fil d'or qu'on trouve sur les couvre-lits à la façon d'Attale ", cf. II 1322 nec sit in Attalico mors mea nixa toro. L'incongru putria provient, je présume, de la condamnation d'un lecteur

${ }^{57}$ Dimundo fait injure à Heinsius en laissant entendre que s'il ne comprend pas le lien entre les deux propositions, c'est qu'il attribue à quippe « il valore di congiunzione causale da collegare a mouerit ". J'ignore à quel usage de quippe se réfère Dimundo; ici on a l'idiomatique quippe et, connu comme tel du ThLL V 2 912,77-78 et dont on rapprochera nam et, Hand, Tursellinus II 508 ss. Heinsius, qui corrige quippe et, n'a peut-être pas tout à fait compris le passage, mais nous verrons qu'il en a mieux vu les difficultés que Dimundo, qui aurait mieux fait de chercher à comprendre où l'illustre érudit voulait en venir.

${ }^{58}$ Comme d'autres commentateurs, Dimundo ne discute pas cet usage pourtant remarquable du subjonctif. Elle ne commente pas non plus iussisses IV 7,30 « tu aurais pu, tu aurais dû demander».

${ }^{59}$ Texta et in ont déjà été trouvés le premier par l'humanisme italien, le second par Heinsius. 
moralisateur et secta... $a b$ résulte peut-être de la mécompréhension de signa, pris au sens qu'a ce mot dans un passage rapproché à mauvais escient par certains commentateurs, Stace Silves III 138 signis crescit torus asper eburnis. L'explication confuse de sine aere par Dimundo 780 dans istius tibi sit surda sine aere lyra (58) me paraît désespérée: je regrette que ni Heyworth ni elle ne mentionnent la conjecture humaniste (cf. Hanslik 1979) retrouvée par Vahlen surda sine aure, pléonasme idiomatique du type sine tegmine nuda ${ }^{60}$. Deux fautes déparent le beau distique Vidi ego odorati uictura rosaria Paesti | sub matutino cocta iacere Noto (61-62): en l'état, uictura ne saurait signifier à lui seul " che parevano destinati a vivere a lungo » et il ne peut provenir du verbe uincere que si l'on restitue odoratum uictura rosaria Paestum (Schippers): les parallèles cités par Dimundo 783 montrent la justesse de la correction qu'elle écarte; ensuite, l'étrange cocta provient du v. 26 murreaque in Parthis pocula cocta focis: Stace Théb. VII 224 dit (rosaria) usta Noto et je gage que Properce avait écrit tosta (ainsi déjà Cornelissen). Après avoir regretté que Dimundo n'accorde pas sa place à crebrescere $\left(\right.$ Housman $\left.^{61}\right)$ pour concrescere $(67)$ - très problématique s'agissant de toux - , relevons la faiblesse frappante de l'épithète paternas dans atque animam in tegetes putrem exspirare paternas. Properce avait peut-être écrit palustres ou palustris: Dimundo cite Festus 444,15 Lindsay scirpus est id, quod in palustribus locis nascitur leue et procerum, unde tegetes fiunt; nous ajoutons Martial XI 32,2 nec tibi de bibula sarta palude teges. Pour ce qui est du v. 72, immundo pallida mitra situ, il suffit d'un coup d'œil à la rubrique afférente de l'article pallidus du ThLL (X1 131,70 ss.) à laquelle renvoie Dimundo pour apercevoir l'hétérogénéité du passage de Properce. La correction squalida (Heinsius) a tout pour séduire (cf. par exemple Sénèque Phèdre 471 orbis iacebit squalido turpis situ; N. Q. V 18,5 ne quid esset situ squalidum; Quintilien, I. O. X,130 arma squalere situ ac robigine).

VI. Fedeli condamne maintenant pelagus v. 17, Actia Iuleae pelagus monumenta carinae ${ }^{62}$, mais défend encore le libellé du vers suivant,

${ }^{60}$ Voir J. Vahlen, Gesammelte Schriften II, Leipzig 1923, 128, 325 et 853.

${ }^{61}$ En observant que Heyworth préfère la forme " plus rare " crebescere, Dimundo ne tient pas compte des observations de F. Bücheler dans son bel article " $R \mathrm{R}$ im Anlaut benachbarter Silben im Latein", NJPHP 105, 1872, 109-19, spécialement 113-116, dont la conclusion suggère que la forme classique était crebrescere. Dans ce cas, la graphie tardive crebescere aura abouti à crescere, qui aura, à son tour, appelé le remaniement métrique concrescere.

${ }^{62}$ Heyworth me paraît avoir tort de préférer son bien faible celebrant à Leucas, l'emendatio palmaris de Markland (PELAGVS est presque un anagramme de LEVCAS). Markland suggère cette correction dans une note critique (p. 307) à un passage de Stace, Silves V 3,154, où $L E V C A D E$ s'est corrompu en CALC(h)IDE, non formidata temeraria Chalcide Sappho (voir mon commentaire critique $a d$ loc.). Là aussi il a pu y avoir une permutation, CALEVDE, qui fit l'objet d'une correction, peut-être animée par l'intention d'introduire un jeu de mots 
nautarum uotis non operosa uia: " i vota dei naviganti possono solo riguardare il felice raggiungimento del porto ", "qui, ovviamente, si tratta del percorso [uia] che la nave deve compiere per raggiungere il porto ", " non operosa ha il senso di 'non faticoso' e quindi, se si considera la litote, di 'agevole'". Je ne comprends pas comment Fedeli peut trouver tout cela éclairé par les vers de Virgile qu'il cite, Én. III 274-275, où il est question de formidatus nautis... Apollo. Je crois bien que ce témoignage et l'épigramme A. P. VI 251 (Philippe) dont Fedeli cite le dernier distique contredisent le vers de Properce tel qu'il est transmis: on attendrait non non operosa mais au contraire operosa, d'où la correction de non en nunc (Carutti, cf. nunc onerata Heyworth), mais nunc est faux, car la dangerosité et le besoin de recourir aux prières ne datent pas d' " aujourd'hui ". Je suggère nautarum uotis exsuperanda uia, "trajet que les marins ne peuvent accomplir/ surmonter que grâce à des prières ». L'expression (au sens d' " achever ") est dans Avienus Orae maritimae I 178-180, Et rursus inde si petat quisquam pede | Tartessiorum littus, exsuperet uiam | uix luce quarta. La graphie de certains mss. est exuperet; chez Properce, exuperanda serait devenu *exoperanda, corrigé en <non> operosa.

En conservant arcus au v. 55, Fedeli inflige à Properce le résultat d'une faute par anticipation: Dixerat et pharetrae pondus consumit in arcus (hostes Heyworth): I proxima post arcus Caesaris hasta fuit. Pour faire échec à la correction évidente furit (Guyet, Heinsius), Fedeli conteste le parallèle de Valerius Flaccus I 144 ense furens au motif que c'est Jason et non l'épée qui furit: cela s'appelle hariolari. Apollon seconde César (Auguste), Rome triomphe de l'Égy pte et César (Jules) constate qu'Auguste est bien son fils: Sum deus; est nostri sanguinis ista fides (60). Telle semble être la paradosis. Le sum deus qu'elle prête à César est d'une fatuité creuse que Fedeli récuse: comme en 1984, il lit Tu deus. Mais est-ce vraiment de divinité qu'il est question ? Est nostri sanguinis ista fides, "cette victoire tienne est la preuve de ton appartenance au même sang que moi ", laisse la divinité hors du jeu. La solution est peut-être Tu meus es (Tu meus Baehrens ${ }^{63}$ ) ou En meus es; nostri sanguinis ista fides (pour En, cf. XI 14 en sum quod digitis quinque legatur onus et, mieux, Ovide Mét. XV 677 En deus est, deus est $\left.{ }^{64}\right)$. Il me semble qu'il y a entre $(t u . .$.$) es et i s t a$, entre meus et nostri

spirituel entre temeraria et $\chi^{\alpha} \lambda_{\kappa \varepsilon o \varsigma}$. Selon Bücheler ap. Fr. Vollmer, P. Papinii Statii Silvarum libri, Leipzig, 1898, 539, le trait d'esprit vient de Stace. Je ne cesse d'être surpris qu'un Wilamowitz (Sappho und Simonides 28-29), se laissant égarer par Vollmer et Bücheler, ait pu défendre Chalcide: ce fourvoiement chez un tel érudit garantit du moins l'immunité à des érudits de moindre envergure qui commettent des erreurs encore plus graves.

${ }^{63}$ Voir Havet, 1916, 122-3. Les deux propositions juxtaposées sont une magnifique illustration du chapitre " Prädikat » du livre déjà mentionné de Brugmann, Die Syntax des einfachen Satzes im Indogermanischen, 57 ss.

${ }^{64}$ En faveur de ce texte, qui est celui de l'édition oxonienne de R. Tarrant (2004), voir H. Magnus, Studien zur Überlieferung und Kritik der Metamophosen Ovids, Fünfter Teil:

ExClass 21, 2017, 175-215 
une correspondance très heureuse. Noter ces potentiels parallèles chrétiens, Augustin Sermo de tempore barbarico VIII (XL 705-706 Migne; paroles $\mathrm{du}$ père au frère du fils prodigue et du Christ à Pierre ) Fili, tu meus es et mecum es semper et omnia mea tua sunt; Rosvith (CXXXVII 1083,11-13 Migne) Post haec intonuit solio uox Patris ab alto, | dicens ad proprium diuino famine Natum: | "Tu meus es charus percerte Filius unus... (V. Rétif de la Bretonne, Poésies latines de Rosvith religieuse saxonne du $X^{e}$ siècle, Paris 1854 126,6-8).

$\mathrm{Au}$ v. 72, blanditiaeque fluant per mea colla rosae, Fedeli a raison d'écarter la leçon " autorisée " blanditiae - à savoir, expliquait-on ${ }^{65}$, blanditiae rosae, gén. sing. = rosa blanda, singulier collectif - , mais la correction qu'il adopte blanditae n'est qu'un pis-aller " paléographique » et j'espère ne pas choquer en disant que l'astuce également " paléographique » blandae utrimque (Lachmann suivi par Heyworth) vaut mieux mais pas beaucoup mieux. Il suffit de citer l'hexamètre précédent, candida nunc molli subeant conuiuia luco, et de se rappeler la dilection de la poésie latine pour le contraste blanc/rouge ${ }^{66}$ pour s'aviser que puniceae (Waardenburgh; puniceae $<$ baniciae < blandiciae = blanditiae ?) a de sérieuses chances d'être ce que Properce avait écrit ${ }^{67}$. Goold saisit l'opportunité que lui donne la correction de Canter irritat pour irritet (75): Heyworth et Fedeli la laissent passer. La façon dont, chez Fedeli (non moins que chez Heyworth), le sujet (79) du verbe reddit et ce verbe (80) sont disjoints, hic referat sero constrictum ${ }^{68}$ foedere Parthum: | "Reddit signa Remi, mox dabit ipse sua..., est très gauche. Cette gaucherie, le fait qu'un hic précédent (78) s'oppose déjà à ille (77) et le fait, trop peu remarqué, que les deux derniers vers paraissent impliquer que les vers 80-84 soient mis dans la bouche de la persona loquens, suggèrent une autre organisation du passage, dont Hutchinson 2006 eut l'idée: Haec referam: "Sero constrictus foedere Parthus | reddit signa Remi, mox dabit ipse sua.

Liber XV, programme du Sophien-Gymnasium, Berlin 1893, 25-6. - Citons aussi, pour ce qui est du vers de Properce, En deus est: nostri sanguinis ista fides de Hanslik, 1979. Ce dernier se trompe en attribuant à Markland et à Lachmann En pour Tu: ils ont suggéré en nostri sanguinis ista fides.

${ }^{65} \mathrm{Ch}$. W. Mitscherlich, Lectiones in Catullum et Propertium, Göttingen 1786, 166 (avec, pour alternative, la correction Paestanaeque). À méditer cette remarque de Mitscherlich au sujet de blanditae, défendu par Fedeli: Nolim tamen Propertio, ut dicam, quae sententiam (lire sentiam), insolentius hoc verbum a magno Scaligero intrusum.

${ }^{66}$ On verra ma note à I $2037-38$.

${ }^{67}$ Voir Verg., ecl. V 17; Hor., carm. IV 10,4 .

${ }_{68}$ Constrictum Hardie ap. Hutchinson, 2006, 169: confessum mss. Le terme technique serait obstrictum foedere. Fedeli a raison de mettre en valeur la conjecture de Hardie. 
VII. S'il est normal que le feu ait rongé le béryl qui rehaussait la main de la défunte Cynthie, il est étonnant que l'eau du Léthé ait usé par frottement ses summa ora: adederat (9) et trita (v. 16) ont peut-être contribué à la substitution de triuerat (10) à tinxerat (Schrader). Dimundo ne s'interroge pas davantage sur triuerat que sur curuum dans Denique quis nostro curuum te funere uidit, | atram quis lacrimis incaluisse togam?(27-28). Pourtant furuum (Passerat, Heinsius) est une bonne idée. Par ailleurs, le mot approprié est non incaluisse, mais inmaduisse, conjecture humaniste qui méritait mieux que d'être citée comme un (prétendu) synonyme plus pauvre de incaluisse. Cynthie reproche à Properce l'absence de ses hommages funéraires; à la place, une rivale se pavane dans une tunique de dessus dont le bas est bordé par une large bande de pourpre et d'or (cyclas): Quae modo per uiles inspecta est publica noctes, | haec nunc aurata cyclade signat humum (39-40). Le contraste entre les deux vers suggère per uiles spectata est publica uestes; inspecta est fait plus difficulté que les commentateurs ne veulent bien le dire. La diction per uestes uiles (instrumental et modal, cf. I 2,2 tenuis Coa ueste mouere sinus; III IX 26 onerare tuam fixa per arma domum) aura surpris ${ }^{69}$ et amené la retouche noctes. Pour l'usage libre de la préposition per (per uestes $=$ in ueste) comparer III 13,29-30 mixta referre | lilia uimineos lucida per calathos; IV 326 det mihi plorandas per tua colla notas. D'après la variante la plus autorisée, la même rivale grauiora rependit iniquis pensa quasillis (41): la vraie leçon me paraît être reponit, " elle place en récompense ", c'est-à-dire en rétribution des propos favorables tenus sur la beauté de leur précédente maitresse par les servantes de la nouvelle (42). Reponit est préférable à imponit (Hutchinson). Cynthie révèle la bipartition des Enfers turpem... per amnem (55): l'évocation des turpitudes qui suit a dû retentir sur l'épithète du fleuve, qui, je crois, était originellement tristem (Burman): Dimundo cite justement Virgile Én. VI 438. Elle lit Andromedeque et Hypermestre sine fraude marita | narrant historiae nota pericla suae (63-64), mais les objections que formule Havet 1916126 contre cette anticipation par narrant de narrat (66) sont considérables et la correction de pectora nota en nota pericla (Heimreich), comme toute correction impliquant le maintien de narrant, doit être retirée. Havet suggère Errant, historiae nota pectora piae, " âmes connues pour une légende pie » et cite III 13,56 pour la confusion pius/tuus.

Le tableautin bucolique Ramosis Anio qua pomifer incubat aruis (81) a légitimement surpris et les justifications tortueuses du texte transmis qu'offrent Heyworth et Dimundo s'évanouissent devant la perfection intrinsèque de la

${ }^{69}$ L'usage propertien de la préposition per est parfois remarquable: Hertzberg (dans le premier tome de son édition, 1843 133) relève III 5,5 per bracchia. Voir aussi Rothstein à I 5,5 et l'index de Butler \& Barber s. v. per. Aucune remarque sur per dans le chapitre pertinent de la monographie de H. Tränkle 1960, aucune entrée per dans l'index de l'édition de Fedeli 1984 et dans l'index du présent commentaire, bien que Fedeli commente per iubas IV 420.

ExClass 21, 2017, 175-215 
correction de Broukhusius et sa plausibilité " paléographique » ou, pour mieux dire, la facilité des fautes qu'elle implique. Broukhusius suggère (verissime et ingeniose Bentley à Horace Odes I 7,14, pulcherrime Lachmann ${ }^{70}$ ) Pomosis... spumifer, conformément à la caractérisation connue de l'Anio ${ }^{71}$ et à l'évocation des pomaria qui le bordent. Dimundo cite Ovide Amours III 6,45-46 Nec te praetereo qui, per caua saxa uolutans, | Tiburis Argei pomifer arua rigas, mais il suffit de consulter l'apparat de l'édition de Ramirez de Verger $\left(2006^{2}\right)$ pour s'aviser que spumifer est une variante de pomifer et que Bentley (au passage d'Horace) défend pomifera (cf. Silius IV 225), avec raison ${ }^{72}$. C'est dans ce paysage idyllique que Cynthie veut que son chantre et amant grave l'épitaphe qu'elle lui dicte: hic (v.1. hoc!) carmen media dignum me scribe columna (83). Dimundo ne dit rien de media; Hutchinson explique "for easy reading and visibility; contrast Luc. 8.821822 ». Mais Properce est un poète, non un épigraphiste, et media paraît ici une précision pédante et malheureuse: lire nitida (cf. Juvénal XIV 60) ? L'épithète aurait de l'à-propos après le rappel du fait que dans le voisinage de Tivoli l'ivoire garde sa blancheur éclatante, (qua...) et numquam Herculeo numine pallet ebur (82). Voici l'un des derniers conseils de Cynthie: $\mathrm{Nec}$ tu sperne piis uenientia somnia portis: | cum pia uenerunt, somnia pondus habent (87-88). Le vers 88 est une très gauche lapalissade et je tiens cum pia pour un affreux bouche-trou: je lirais his (ou hac) quae uenerunt, somnia pondus habent, " les songes qui passent par ces portes, (ceux-là seuls) sont substantiels".

VIII Le détour à Lanuvium, pour voir le rituel local du draco, vaut la peine: tam rarae non perit hora morae (4). Gratae (Housman) est écarté par les éditeurs mais paraît s'imposer. Fedeli a raison de mettre entre croix le second hémistiche tale iter omne cauet (6) - que la ponctuation uirgo, tale iter omne caue n'améliore pas - et de ne pas trouver entièrement satisfaisante la conjecture placée par Housman en marge de son exemplaire de l'édition de Baehrens 1880 et adoptée par Heyworth, tale iter omen habet. C'est " paléographiquement " astucieux mais, pour le sens, creux. Élien $(N$. $A$. XI 16) rapporte un détail non dénué d'importance et absent du texte transmis de Properce, à savoir qu'un bandeau empêche les jeunes filles d'y

\footnotetext{
${ }^{70}$ Voir aussi la remarque, cruelle pour ceux qui défendent, voire même exaltent, la paradosis, de Housman Classical Papers 1237.

${ }^{71}$ Voir P. Burman à Ovide Amours III 6,46, Amsterdam 1727, I 492.

${ }^{72}$ Dimundo 520 rapproche de IV 3,4 haec erit e lacrimis facta litura meis « l'antecedente letterario del topos ", à savoir Catulle LVIII 2, conscriptum hoc lacrimis mittis epistolium, mais le topos (et le bon sens) réclame conspersum (Schrader), ce qui n’a échappé ni à Baehrens dans son commentaire de Catulle, 492, bien qu'il préfère sa propre et regrettable correction constrictum e lacrimis, ni à J. M. Trappes-Lomax, Catullus: A Textual Reappraisal, Swansea $2007,227-8$.
} 
voir. L'hémistiche corrompu était-il relatif à cela ? Dans ce cas, je suggère la parenthèse (taenia lumen habet). La Phyllis qui consolera le locuteur est sobria grata parum: cum bibit, omne decet (30), c'est-à-dire, explique Fedeli, soit omne decet in illa, "when she drinks, all charm " (Heyworth), soit omne decet ei (lire eam), " elle se permet tout ". Le contexte me paraît nettement en faveur de la première interprétation, qu'exprimerait mieux omne decus, tour bien connu (qu'il suffise ici d'invoquer Virgile Buc. V 34 tu decus omne tuis). Il est regrettable que Fedeli n'adopte pas le certain discubitus (Palmer) pour concubitus (36) et qu'il ne mette pas entre croix Nile, tuus (39). La table qui tombe sur ses pieds, reccidit inque suos mensa supina pedes (44), est un non-sens: il y a peut-être là un cas intéressant d' " erreur polaire ", inque suum... caput (Heyworth). Le locuteur fait la fête, présent de corps mais absent d'esprit, car il pense à Cynthie, qui fait une irruption fracassante: Lanuuii ad portas, ei mihi, totus eram, I cum subito rauci sonuerunt cardine postes (48-49). Je ne comprends pas pourquoi Fedeli met un point entre eram et cum. C'est bien connu que, dans la figure de grammaire du cum inuersum, l'opposition des durées peut être mise en valeur par un adverbe du sens de subito, mais ici il manque un mot rattaché à cardine: je suggère que subito s'est substitué à moto (cf. Ovide Amours I 6,2 moto cardine pande forem, 49 uerso sonuerunt cardine postes). Nec mora, cum totas resupinat Cynthia ualuas (51), lisent les manuscrits, mais totas est bizarre et la phraséologie juste serait contortas... ualuas (cf. Valerius I 609): dans les deux autres occurrences de nec mora (IV 84; X 36), il y a parataxe et cum est absent. Le locuteur décrit sa réaction de surprise avec pittoresque: Pocula mi digitos inter cecidere remissos | pallueruntque ipso labra soluta mero (54-55). Soluta ne fait pas sourciller Fedeli; je soupçonne qu'il y a faute par anticipation de solutis v. 60 et/ou par permutation de lettres et que Properce avait écrit lutosa "barbouillées " (cf. Stace Silves IV 939 dulci defruta uel lutosa caeno). L'arrivée brutale de Cynthie fait pâlir les lèvres du locuteur rougies par le vin: c'est juste le contraire des rosea labella du Gellius de Catulle LXXX, qui deviennent hiberna candidiora niue parce qu'elles sont emulso (labra) notata sero. Les fureurs de Cynthie réveillent tout le quartier, mais Fedeli croit encore à la leçon transmise lumina dans lumina sopitos turbant elata Quirites (58): certes, il a raison de critiquer le très " paléographique " Crimina adopté par Heyworth, mais il aurait dû s'intéresser à Iurgia de Baehrens. Fedeli aurait au moins dû discuter la variante cui, qui remédie au lien défectueux que constitue cum (72): si Heyworth avait, comme Goold, adopté cui, cela aurait du moins sensibilisé Fedeli à la diffculté. Fedeli se bat inutilement les flancs pour défendre riserat (82): on ne peut faire sans la restitution du parfait (risit et Heyworth).

On considère ordinairement la conclusion (réconciliation dans le lit), Atque ita mutato per singula pallia lecto | respondi, et toto soluimus 
arma toro, comme gâchée par la faute respondi, qui répète respondi du v. 81 . Fedeli trouve bon de défendre respondi " sinonimo di satisfeci, ovviamente in senso erotico ». Mais les vers qui précèdent n'expriment aucune " exigence sexuelle » de Cynthie et Vahlen (Gesammelte Schriften II 125), dont Fedeli invoque la défense de respondi, n'a en vue que le commandement de Cynthie relatif au ménage et à la purification. Le distique conclusif n'est, à notre avis, plus relatif à ces derniers. Non seulement nous pensons que respondi est gâté mais nous étendons le soupçon à toto (noto Heinsius, laissant de côté, à juste titre, III 31 tum queror in toto non sidere pallia lecto) et surtout à mutato per singula pallia lecto. Que vient, en effet, faire cette évocation alambiquée et même, nous semble-t-il, étrange du changement du lit "d'un drap l'autre ", " drap par drap ", " drap après drap ", dans la scène où les deux amants se réconcilient en faisant l'amour ? Soluimus arma indique à la fois la résolution du conflit et l'orgasme qui clôt les ébats. Je crois deviner, sous mutato per singula pallia lecto, qu'a peut-être suggéré la variante et totas iterum mutare lacernas (85), l'évocation de ces ébats: turbato per singula proelia lecto, " après que nos ébats successifs eurent mis le lit en désordre » (cf. II 1,45 angusto uersamus proelia lecto, et, sur les souffrances du lit d'amour, comparer Catulle VI 9-11; Horace Épodes XII 11-12 iamque subando I tenta cubilia tectaque rumpit; Properce II 29,35-36; Ovide Amours III 14,26). Respondi et nous paraît avoir pris la place d'un participe indiquant la fatigue amoureuse, defessi (cf. Epistula Sapphus 49-50). Au total, Atque ita turbato per singula proelia lecto | defessi noto soluimus arma toro, nous semble former une conclusion plausible, digne, pour le sens et le style, du poème et de son auteur.

IX. Tite-Live I 7,5 présente Cacus comme pastor accola eius loci et il faudrait se demander si cela ne corrobore pas le remplacement de incola (9) par accola, conjecturé ici par Schrader, dont j'ai accepté la même correction chez Valerius Flaccus IV 513. La ruse de Cacus n'échappa point: nec sine teste deo furtum (13), lit Fedeli 1133-1134 d'après Heyworth pour furem, qui, joint au second hémistiche, contribuait à former une jolie anaphore, furem sonuere iuuenci, | furis et implacidas diruit ira fores, et une expression frappante mais tout à fait intelligible et latine, furem sonuere «indiquèrent le voleur en mugissant». Rothstein explique et illustre le tour et les objections que formule Hutchinson portent à faux. Furtum crée d'ailleurs une ambiguité fâcheuse, car pourquoi comprendrait-on plutôt nec sine teste deo furtum plutôt que furtum sonuere iuuenci ? Fedeli rapproche très bien Ovide Fastes I 560 mugitum rauco furta dedere sono. Au lieu de mettre un point après le v. 12 , il faut ponctuer ainsi:

auersos cauda traxit in antra boues, nec sine teste deo: furem sonuere iuuenci... 
Nec sine teste deo est une correction qui s'ajoute à la proposition précédente, comme souvent nec sine causa ou d'autres tours avec nec sine. Fessos.... boues (4) suggère que Heyworth a raison de substituer quaesiti à quaesitae (18) gardé par Fedeli. Ce dernier (1140) invoque, après Hutchinson 210 , les vaches de la tradition grecque, " che usa sempre il femminile per la mandria di Gerione »: " in der Geryonessage (...) die rinderherden (denn die stiere fehlen mit nichten, so dass es falsch, wenn auch für die vergleichung mit der morgenröte erwünscht ist, mit kühe zu übersetzen) gehören dem riesen ", écrivait Wilamowitz en combattant le naturalisme indo-européen de certains indologues ${ }^{73}$. Goold, Heyworth, Hutchinson et Fedeli conservent feta au v. 22: terraque non ullas feta ministrat aquas (sc. sitienti Herculi). Fedeli explique que feta est oppositif, quamuis feta, et que "il suolo su cui sorgerà Roma viene assimilato a una dona incinta, che però non riesce a partorire ". Mais le sens oppositif paraît faux: la terre ne peut désaltérer Hercule parce qu'elle est gravide et n'a pas encore mis bas ses eaux. Cela reste bien obscur. Hutchinson agite le deus absconditus (ou ex machina) qu'est, pour l'exégèse moderne de Properce, Callimaque, chez qui « Rhea bids the earth 'give birth to' $(H y .1 .29)$ water in a still riverless Arcadia ». Hertzberg 1845488 invoquait déjà Callimaque, mais sans envisager d'allusion cryptique. Le problème est - Hutchinson le voit - que Properce semble alors « ignorer l'irrigation mentionnée v. 5-6 et IV 4,3-6 ». La terre, grosse, aurait donc déjà engendré des eaux, mais Properce aurait oublié ce qu'il vient de dire. À moins que le poète ne fasse obscurément allusion à des fontaines qui existaient de son temps et non de celui d'Hercule, il y a peut-être lieu de considérer la possibilité d'une faute. Le v. 31, que nous discuterons, illustre, remarque $\mathrm{H}$. $\mathrm{Keil}^{74}$ avec sagacité, la sécheresse de la terre, que mentionne Apollonios de Rhodes I 1147-1148 dans un contexte qu'il juge voisin (il s'agit du Dindyme toujours sec avant l'apparition merveilleuse d'une source). Ajoutons exhausto iam flumine uicerat aestum (64) ${ }^{75}$ Keil suggère donc tosta, quitte à lire torquet (ainsi Fedeli) et non torret (ainsi Heyworth) au vers précédent pour éviter la répétition. Vsta (Palmer) serait préférable; comparer Sénèque Q.N. IVa 2,1 quo maxime usta feruoribus terra undas (Nili) altius traheret. Shackleton Bailey 1956 258-259 me paraît avoir encore égaré Fedeli en défendant lucus dans le vers qui évoque le bois sacré où Hercule cherche à se désaltérer, lucus ubi umbroso saepserat orbe nemus. La correction de Fontein saepserat, acceptée par Fedeli, pour fecerat rend lucus encore plus choquant et murus (Fontein) encore plus attrayant. Fedeli n'a donc accompli que la moitié du chemin: murus était pourtant à portée. Il faut

${ }^{73}$ Euripides, Herakles $1909^{2}$ IX.

${ }^{74} \mathrm{H}$. Keil , Observationes criticae in Propertium, Bonn 1843, 18.

${ }^{75}$ West, Indo-European Myth and Poetry, 262 rapproche la version laquelle Cacus est Garanus et Grannus dieu celte de l'eau et des endroits arrosés. 
peut-être aussi accorder plus d'attention aux corrections pénétrantes de Fontein casta pour clausa et uerendos pour piandos (25) et à son densis ou, mieux, à glaucis (Housman) pour longis (29). D'après le texte transmis, Hercule aborde l'enceinte du bois sacré in siccam congesta puluere barbam (31), " eine seltsame Vorstellung ", dit Rothstein, qui inspira à Housman une correction malheureuse, huc in sicca ruit congesta puluere labra, pour huc ruit in siccam congesta puluere barbam. Heyworth (Cynthia 488) rencontre peut-être le vrai en améliorant siccam faucem de Baehrens en siccas fauces: comparer sicco... palato (21) et Lucain IX 503 squalebant puluere fauces, à propos des soldats assoiffés dans le désert libyen. La prêtresse éconduit le héros avec ces mots, Parce oculis ${ }^{76}$, hospes, lucoque abscede uerendo (53). Comme Hercule est resté aux portes et ne rentrera par effraction que plus tard, abscede n'est pas juste et absiste (Markland), " reste à l'écart ", pourrait bien être ce que Properce avait écrit: rapprocher Virgile Én. VI 659 totoque absistite luco. Hercule se venge de l'affront subi en interdisant aux puellae l'accès de l'Ara Maxima, Herculis Inuicti ne sit inulta sitis (70). Inuicti est une correction de Burman très audacieuse, éloignée des données de la tradition (cf. exterminium NFL !): pour être moins contestable, elle devrait offrir un sens parfaitement adéquat, mais, si elle restitue la phraéologie " officielle $"{ }^{77}$, cette épithète fait perdre le lien avec l'étiologie de la fondation de l'Ara Maxima. Externi (Heinsius, suivi par Heyworth), "étranger " (car il s'agit de l'Hercule grec), a le mérite de se tenir au plus près possible de la paradosis ${ }^{78}$. Le lien "étiologique » serait clairement sauvegardé avec exclusi (Owen). Le lexique de l'exclusion est prépondérant dans le poème et, de surcroît, exclusi mettrait en relief une étymologie de Hercules évoquant celle que suggéra un temps Mommsen, " *hercere [mot

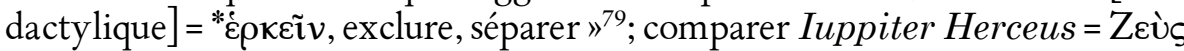

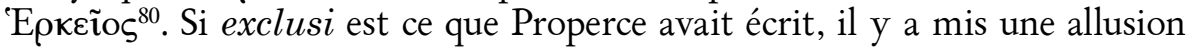
à l'état d'amator exclusus qu'il prête à son propre personnage ${ }^{81}$. Il est vrai

${ }^{76}$ Fedeli 1177 semble reprocher à Rothstein son explication " in parce oculis liegt nur das Verbot die Augen in Tätigkeit zu setzen ", mais c'est bien le sens de l'expression ici, comme celui de parcite luminibus chez Tibulle I 2,35. Il n'est pas question de "risparmiare la vista ».

${ }^{77}$ Wissowa, Religion und Kultus, 273.

${ }^{78}$ Je ne comprends pas comment H. C. Günther (Brill's Companion to Propertius, Leiden 2006, 388 n. 170) peut écrire "Richmond's Oestrumni (cf. Avien. Or. Mar. 90) has also [par rapport à externi] a good chance to be correct ». Il n'en a rigoureusement aucune (cf. Butler \& Barber).

${ }^{79}$ Je traduis Th. Mommsen, Die unteritalischen Dialekte, Leipzig 1850, 262.

${ }^{80}$ Wissowa, Religion und Kultus, 119 n. 4. Première occurrence dans la poésie latine Ovide Ibis 284. Selon M. Paschalis, Virgil's Aeneid: Semantic Relations and Proper Names, Oxford 1997, 147 et 292, Virgile rattache Hercules à ह̌pкоs.

${ }^{81}$ W. S. Anderson, "Hercules Exclusus: Propertius, IV, 9", AJPh 85, 1964, 1-12 exploite le thème à fond. Il fait (p. 10 n. 23) l'éloge de exclusi, que néanmoins il rejette " reluctantly ", apparemment à cause de son éloignement par rapport à exterminium, qui, lui fait remarquer un collègue, oriente vers externi $<$ extermi $<$ extermi<nium $>$. Certes, mais est-on sûr que le 
qu'on pourrait aussi tirer externi vers le sens de exclusi, avec un double-sens, " étranger » et " qui reste à l'extérieur ", donc " exclu ». Nous avons discuté plus haut la " conclusion " du poème (71-74), en laquelle nous proposons de voir une interpolation.

X. Dans la caractérisation de Romulus, telle qu'elle est transmise et acceptée par Ciccarelli, Idem eques et frenis, idem fuit aptus aratris, I et galea hirsuta compta lupina iuba (19-20), on attend equus (equos Housman), ce qui a l'inconvénient de rendre plus difficile la connexion par et devant galea, qui se rapporte à Romulus. Hutchinson 2006225 remarque que idem... idem est absent de la poésie augustéenne et suggère de remplacer soit le premier idem soit et (devant frenis) par huic; dans ce cas, la connexion par et (v. 20) est facilitée et on peut lire sans remord equus ou equos. Pour le très idiomatique et (frenis) " non répété ", Butler \& Barber comparent Ovide Fastes VI 224, utilis et nuptis, utilis esse uiris. Ciccarelli cite Pline le Jeune Lettres VII 4,6, dont la pertinence m'échappe totalement. Le ThLL V,2 887,32-35 mentionne justement Ovide Mét. I 54 illic et nebulas, illic consistere nubes iussit, et V 612 per tamen et campos, per opertos arbore montes ${ }^{82}$. Ces passages montrent que le $e t$ " non répété " s'accompagne de la répétition d'un mot: il faut donc garder idem... idem et se résigner à sousentendre le huic introduit à tort par Hutchinson.

Je m'étonne que Ciccarelli puisse défendre ossibus dans in uestris ossibus arua metunt (v. 30, à propos de l'antique Véies abandonnée) en invoquant Ovide Hér. I 51-56, car il y a un monde entre le vers de Properce et semisepulta uirum curuis feriuntur aratris | ossa. La solution la plus plausible est peut-être sedibus (Phillimore). Je tiens pour absolument impossible en soi et indigne de Properce le libellé transmis, et accepté par Ciccarelli, des v. 31-32, Forte super portae dux Veiens astitit arcem । colloquiumque sua fretus ab urbe dedit. En combinant des corrections adoptées ou citées par Heyworth, on obtient une version plausible, Forte

point de départ supposé, externi, est la vraie leçon ? Le répertoire de Smyth 1970 omet la bizarre conjecture d'Anderson Herculeum exitium ne sit inulta sitis, "Let not thirst unavengend be the ruin of Hercules".

${ }^{82} \mathrm{Il}$ me semble que le rédacteur de l'article du $T h L L$, J. B. Hofmann, se fourvoie en rapportant $e t$ chez Ovide et Properce à et conjonctif et non à et adverbial. J. Svennung, Untersuchungen zu Palladius und zur lateinischen Fach- und Volkssprache, Lund 1935492 discute (sic)ut et... ita, (sic)ut... ita et et compare l'emploi du grec koi (répété ou présent une fois soit dans la protase soit dans l'apodose) dans les structures comparatives (cf. Hofmann-Szantyr 483, "Zusatz »). Dans cet emploi, il s'agit bien sûr de l'adverbe, non de la conjonction; il en va de même, à mon sens, dans les passages d'Ovide et de Properce où la comparaison, pour être paratactique et non syntactique, n'existe pas moins: equus sicut et frenis, ita aratris aptus fuit. Il ne faut pas oublier que le sens primitif de kai est « comme » (Brugmann, Grundriss der vergleichenden Grammatik II 1, Strasbourg 1890,791) et que le sens originel de et (op. cit. I Strasbourg 1886, 276) est « encore». 
super portam dux Veiens astitit arcis | colloquiumque astu fretus ab hoste petit. Il est regrettable que Ciccarelli refuse la correction toute simple fortis dans l'énergique phrase forgée par Cossus, Forti melius concurrere campo (35).

Ciccarelli 1251 fait valoir la célèbre mention du triomphe de Marcellus de Galleis Insubribus et Ger[man(eis) des Fastes Triomphaux pour défendre Rheno au v. 39, Claudius at (a mss.) Rheno traiectos arcuit hostes. Karl Müllenhoff, Deutsche Altertumskunde II, Berlin 1887, 195 veut que le rédacteur des Fastes et Properce aient été victimes de la substitution, par le fait des annalistes de l'époque syllanienne et d'après, des Germani aux Gaesatae et du Rhin au Rhône, "wie es scheint aus keinem andern grunde, als um des reizes der neuheit willen ». Mais Properce (42) associe à Virdomar gaesa, le dard gaulois éponyme des Gaesatae ${ }^{83}$, et cela paraît coller non avec Rheno mais avec Rhodano ou Rhodanum, hydronyme restitué par Postgate. Ciccarelli emprunte à Fedeli 1984 son texte, à mon avis très gauche et même flirtant dangereusement avec l'inintelligibilité, du v. 43, Illi uirgatis iaculanti ante agmina bracis | torquis ab incisa decidit unca gula. La supériorité du texte conjectural (Schrader et Waardenburgh) adopté par Heyworth, illi uirgatas maculanti sanguine bracas, me paraît frappante. Il reste que la difficulté de unca, relevée par Havet 1916 129-130, échappe à Heyworth et Ciccarelli, qui traduisent comme si unca signifiait tortilis (" twisted ", " ritorta "). Havet suggère torquis ansa, " la courbe pendante formée sur la poitrine par la partie antérieure du collier »: " cessant d'être soutenue d'un côté, l'ansa, seule visible pour le regard, " tombe ", c'est-à-dire devient verticale et rectiligne, puis ce qui la formait glisse, en entraînant la partie cachée derrière le coup ". Qui eût pu comprendre ansa sans ce commentaire trop précis ? Je tente uda ou uncta, "imbibé (de sang)", en rapprochant le passage de Claudius Quadrigarius cité par Ciccarelli, caput praecidit, torquem detraxit eamque sanguinulentam sibi in collum imponit. Les quatre derniers vers souffrent de défauts qu'il suffira ici de signaler en substituant aux leçons suspectes des corrections plausibles ou possibles: Haec (Colucio Salutati, Nunc ou Nec mss.) spolia in templo tria condita: causa Feretri | illast(Lib., omine mss.) quod certo dux ferit ense ducem; I seu, quia uicta suis umeris huc (Broukhusius, haec mss.) arma ferebant, | hinc Feretri dicta est ara superba Iouis.

XI. Les vers 3-4 et 7-8 souffrent du refus des conjectures sedes (Heinsius) pour leges (v. 3), fores (Heinsius) pour uiae (v. 4) et inuida (Boot) Parca (Markland et indépendamment Havet) pour lurida porta (v. 8), où porta est une "Perseverationsfehler " due à portitor du vers précédent. Le texte ubi portitor aera recepit, obserat umbrosos lurida porta locos est, à mon

${ }^{83}$ Vor Müllenhoff, 206, note. 
sens, une offense faite à la poésie en général et à Properce en particulier. Nous avons examiné plus haut le cas des v. 5-6. Une crux célèbre dépare les vers 15-16: Damnatae noctes et uos, uada lenta paludis, I et quaecumque meos implicat unda pedes. Il est instructif de le constater: de même que Fedeli a cessé de défendre uada lenta, paludes (mss., paludis Santen), de même il ne soutient plus damnatae noctes, pour la justification duquel il s'appuyait jadis, hélas, sur Shackleton Bailey 1956. Fedeli 1303 réclame la restitution d'un élément « che (...) si colloca allo stesso livello della $\lambda i ́ \mu \nu \eta$ 'A ¿epovoía " et écarte, entre autres, le texte heyworthien damnatae nocti sedes, uada lenta Acherontis. Cet élément dont Fedeli regrette l'absence doit être distinct des uada lenta paludis, sans quoi et uos ne se comprend pas. Fedeli ne suggère rien lui-même pour remplacer les Damnatae noctes que lui-même condamne: Flammati fontes (le Phlégéthon) pourrait-il faire l'affaire ? Fedeli critique à juste titre la substitution par Heyworth de aut à et devant quaecumque: il me paraît certain qu'il y a de et uos (qui disparait chez Heyworth au profit de la conjecture damnatae nocti sedes) à et quaecumque une suite graduée aussi poétique qu'idiomatique: comparer III 21,15-16, Romanae turres et uos ualeatis amici, | qualiscumque mihi tuque, puella, uale. Ce parallèle suggère que sous damnatae noctes pourrait se dissimuler un élément différent de celui qu'appelle Fedeli, par exemple Taenariae fauces (Géorg. IV 467). Les juges de Cornélie seront Éaque et ses frères Minos et Rhadamanthe: assideant fratres, iuxta et Minoida sellam | Eumenidum intento turba seuera foro (21-22). Fedeli commente à loisir intento foro, notation dont la pertinence peut être mise en doute; ce doute et le mouvement de la phrase ainsi que la ponctuation même adoptée par Heyworth et Fedeli (virgule après fratres) suggère Eumenidum intendat/ intentet turba seuera faces (intendat Graevius, intentet faces van Eldik). Intentet... flagrum semble une possibilité; intento... flagro serait plus proche mais ne règlerait qu'une difficulté.

Les v. 34-35, Mox, ubi iam facibus cessit praetexta maritis, | uinxit et acceptas altera uitta comas, font l'objet d'explications peu claires, non moins sommaires que hâtives, chez les commentateurs de Properce. Fedeli ne fait nullement exception. « Fino alle nozze, explique-t-il (1328-1329), le giovani portavano i capelli raggruppati dietro le spalle grazie ad un'unica fascia (cfr. Val. Fl. 8,6), mentre il giorno delle nozze li dividevano in più trecce (...). L'espressione tecnica per designare l'acconciatura di una donna sposata (capere crines: cfr. Plaut. Most. 226), viene qui variata in accipere comas, anche se Properzio parla solo di una seconda (altera) fascia in luogo delle sei, con le quale secondo Festo (454,23-24 L.) nubentes ornantur (cfr. anche Varr. ap. Non. 353,26-28 L. e Marquardt 1879, 38) ». Fedeli paraît confondre deux choses à distinguer, les sex crines, six tresses ou nattes, de la mariée et la « bandelette » de la mariée, qui 
s'oppose à celle dont la jeune fille noue ses cheveux. Marquardt ${ }^{84}$ suppose qu'il s'agit d'une " bandelette double " mais Graillot ${ }^{85}$ a peut-être raison de penser que altera uitta désigne " l'autre bandelette ", celle de la mariée en opposition à la bandelette virginale (Valerius Flaccus VIII 6, ultima uirgineis tum flens dedit oscula uittis [pluriel poétique]). On pourrait concilier ces sex crines et l'altera uitta en supposant (par exemple) que le bandeau maintient les six nattes ${ }^{86}$. Les exégètes modernes admettent sans sourciller que acceptas comas adapte le tour technique capere crines, "se coiffer en nattes ", mais comas n'est pas crines, qui est ici technique, et, comme épithète de comas, acceptas est, en admettant d'y voir l'équivalent de captos (crines), d'une extraordinaire faiblesse. Qui a assez peu de sentiment de la langue et du style des poètes pour admettre que, parce que capere crines vaudrait " se marier ", acceptas comas pourrait signifier " la coiffure de la mariée " ? Avec cette faiblesse contraste la pertinence de l'expression dans le passage de Plaute que citent Marquardt et Fedeli: Si tibi sat acceptumst, fore tibi uictum sempiternum Atque illum amatorem tibi proprium futurum in uita, Morem gerendum censeo tibi et capiundas crines. Il me paraît difficile de nier que dans le passage de Properce comas appelle une épithète descriptive, comme l'a bien compris Juste Lipse dans une note à Tacite Ann. III 53. Il se prononce pour aspersas, leçon qu'il croyait autorisée mais qui n'est qu'une conjecture, et une conjecture dont le sens ne paraît guère convenir. Adstrictas (Heinsius) aurait pour lui le parallèle de Tibulle I 6,67-68, quamuis non uitta ligatos | impediat crines. D'autres textes que cite Marquardt 1891, 55 n. 1 suggèrent innexas, intortas (voir aussi Valerius VI 563, Silius III 284; contortis comis chez Properce IV 7,80) ou encore implexas, implicitas. On objectera que la corruption de intortas, que je privilégie, en acceptas n'est guère plausible; peut-être tempérera-t-on ce jugement si l'on veut bien considérer la possibilité que chez Horace, carm. saec. 62 (acceptusque nouem Camenis), acceptus soit une leçon fautive pour insertus ${ }^{87}$ et la possibilité que, chez Properce lui-même (I 6,34), accepti se soit substitué à $a s s e r t i^{88}$. Incinctas mérite peut-être d'être proposé (cf. Ovide Fastes V 337 ebrius incinctis philyra conuiua capillis); noter que, pour acceptus chez Horace, M. D. Reeve m'a suggéré accinctus, que j’avais moi-même écarté au motif peut-être non décisif que le sens de accinctus qui conviendrait au passage est plus tardif. Mais intortas « tressés " paraît plus adéquat.

${ }^{84} \mathrm{~J}$. Marquardt, La vie privée des Romains, ouvrage traduit sur la deuxième édition allemande avec l'autorisation de l'auteur par V. Henry I, Paris 1891, 56 n. 1.

${ }^{85}$ Dictionnaire de Daremberg et Saglio, article Vitta, V 950A n. 9.

${ }^{86}$ Comparer August Rossbach, Untersuchungen über die römische Ehe, Stuttgart 1853, 287; A. Gallia "The Vestal Habit”, CPh 109, 2014, 225-6 (avec reproduction d'un portrait parlant mais sans analyse précise du passage de Properce).

${ }^{87}$ Voir Annuaire de l'École pratique des hautes études, Section des sciences historiques et philologiques 147, 2016, 104-105.

${ }^{88}$ On verra la note de notre commentaire critique ad loc. 
Fedeli illustre, avec des parallèles qui ne sont pas tous justes, censurae legem mollisse (41) et joue avec l'idée ingénieuse que Cornélie indique à ses juges qu'elle n'a pas cherché à influencer son censeur d'époux " in modo da coprire sue eventuali colpe ", mais c'est, me semble-t-il, une manière douteuse d'affirmer son comportement vertueux que de dire qu'on n'a pas tenté d'atténuer la rigueur de la loi, et la suite, neque ulla | labe mea nostros erubuisse focos, suggère que Cornélie jure plutôt ne pas avoir contrevenu à la loi, ce qui serait uiolasse (Heinsius), assez proche de mollisse (amené par mollia iura v. 18 ?). Aux v. 47-48, Mi natura dedit leges a sanguine ductas, I nec possis melior iudicis esse metu, la seconde personne généralisante tombe sous le coup du grief de frigiditas, si j'ose dire. Il me paraît très probable que Cornélie a recouru, dans la continuité de $m i$, à la première personne, beaucoup plus pathétique: « et la crainte de la sanction n'aurait pu me rendre plus vertueuse », ce qui suppose possem (cf. ne possem dans les dett.) ou potui (Peerlkamp), correction qui pourrait paraitre plus élégante mais qui est plus loin du texte transmis et souffre d'une ambiguité fâcheuse, car potui pourrait en théorie signifier « je ne suis pas parvenue à être plus vertueuse avec la crainte de la sanction "! Au v. 59, Ille sua nata dignam uixisse sororem | increpat, "Auguste se lamente qu'est avec moi disparue une sœur vraiment digne de sa propre fille ", l'élégance suggère de lire ille sua dignam nata ${ }^{89}$. Si son époux la remplace, seu... sederit et nostro cauta nouerca toro (85-86), Cornélie engage ses enfants à supporter le remariage de leur père: coniugium, pueri, laudate et ferte paternum (87). Quand on voit le mot laudate au v. 89, on s'avise aisément qu'il y a "Antizipationsfehler" et que sous le premier laudate se cache <dur>ate (Housman). Fedeli ne mentionne même pas cette conjecture. Il commente ingénument cauta, mais le sens, la phraséologie et la représentation stéréotypée de la belle-mère (cf. 89-90 !) appellent incontestablement une autre épithète, torua (Heinsius), nostrotorua $<$ nostorua $<$ nostro $<c a>u<t>a$. Si la nouerca était cauta, pourquoi Cornélie demanderait-elle à ses enfants de supporter la nouvelle femme de leur père et pourquoi les assurerait-elle que, s'ils se montrent aimables, leur belle-mère sera apprivoisée, capta dabit uestris moribus illa manus (v. 88) ? On répondra que ferte signifie efferte, " portez aux nues", et que laudate et ferte et cauta sont compatibles et même se corroborent, mais qui peut sérieusement imaginer que Cornélie non seulement envisage une belle-mère cauta mais va jusqu'à prier ses enfants de porter aux nues la nouvelle union de son mari ? Même le " approvate e accettate " de Fedeli, en admettant cette dévaluation du sens de laudate (opposer le sens de laudate, v. 89) ne donne pas satisfaction. De toute façon les vers 88-90 corroborent

${ }^{89}$ Passage omis par D. R. Shackleton Bailey, Homoeoteleuton in Latin Dactylic Verse, Stuttgart/Leipzig, 1994 82. Il cite I 13,32 illa suis uerbis cogat amare Iouem, où aucune transposition n'est possible.

ExClass 21, 2017, 175-215 
l'image d'une nouerca non cauta (étrange épithète), mais torua. Fedeli ne discute pas torua. Si Heyworth avait adopté torua et durate, on les retrouverait peut-être dans le texte de Fedeli. De là l'intérêt d'adopter des conjectures probables: on en signale ainsi mieux la valeur à ses successeurs qu'en les reléguant à l'apparat critique.

G. LiBERMAN

Université Michel de Montaigne Bordeaux III gauthier.liberman@orange.fr 
